\title{
Development of a Physics-Based Combustion Model for Engine Knock Prediction
}

\author{
U.S. Department of Energy \\ Energy Efficiency and Renewable Energy \\ DE-FOA-0001201: FY2015 Vehicle Technologies Program Wide Funding \\ Opportunity Announcement \\ Final Scientific/Technical Report \\ February 1, 2016 - March 31, 2020 \\ Award Number: DE-EE0007334 \\ Award Type: Cooperative Agreement
Prime Recipient: The Ohio State University
Prime Recipient Type: University
DUNS: 832127323 \\ Phone: (614) 688-2116 \\ E-mail: kim.5061@ osu.edu \\ Principal Investigator (PI): Seung Hyun Kim \\ FFRDC Partner: Oak Ridge National Laboratory \\ PI: Derek Splitter
}

Date: June 25, 2020 


\section{Summary}

The objective of this project is to improve the prediction of engine knock by developing a new combustion modeling framework. Engine knock is a limiting factor to constrain the increase of fuel efficiency for spark ignition (SI) engines in most passenger cars. Efforts to increase fuel efficiency, increasing the compression ratio or downsizing, lead to the increase in the tendency of the knock occurrence. The knock is an undesired ignition of the end-gas, unburned fuel/air mixture ahead of the spark-ignited premixed flame, resulting in rapid in-cylinder pressure rises and engine damages.

The combustion modeling framework developed in this project can consider turbulence-chemistry interactions during end-gas ignition, while using a reasonably detailed chemical mechanism developed for ignition and combustion reactions under engine relevant conditions, and the subtle characteristics of spark-ignited flame propagation. It is developed in the context of large eddy simulation (LES), which can capture stochastic in-cylinder processes. The developed model is incorporated into a commercial software for engine simulation, CONVERGE CFD, as a user defined function, and validated. Engine knock and knock-free experiments as well as direct numerical simulation (DNS) of end-gas ignition in homogeneous turbulence are performed to help model development and provide data sets for model validation. With further validation, the developed model is expected to advance the predictive capability for engine knock simulations and thus contribute to improving the fuel efficiency. 


\section{Goals/Objectives and Accomplishments}

The ultimate goal of this project is to improve the capability of computational fluid dynamics in predicting engine knock phenomena. Toward this goal, it was proposed to develop a new combustion modeling framework for large eddy simulation (LES) of engine knock processes with the focus of the development being placed on the following specific aspects:

- Effects of turbulence on end-gas ignition

In typical engine conditions, in-cylinder flows are highly turbulent. Complex turbulent motions substantially affect heat release rates and end-gas ignition. The physics-based and accurate modeling of such turbulence-chemistry interactions is necessary, in particular to predict processes in high scalar dissipation layers.

- Detailed chemistry description

Accurate description of end gas ignition requires the use of a detailed chemical mechanism. This project aims to enable engine knock LES where ignition chemistry is described by a reduced mechanism that contains about 100 species, while employing a physics-based model for turbulence-chemistry interactions.

- Validation with high-fidelity knock experiments

For thorough validation, engine knock and knock-free experiments coupled with direct measurements and chemical characterization of in-cylinder gas species temporal evolution are to be performed to provide high-fidelity data sets. The laboratory experimental data is complemented by direct numerical simulations (DNS), which serve as numerical experiments to provide detailed statistics necessary to assess some specific aspects of the developed model.

The proposed tasks were:

Task 1.1: Development of Base Modeling Framework

Task 1.2: Experimental Campaign I

Task 1.3: DNS of End-Gas Ignition

Task 2.1: Knock Model Development: Validation with DNS

Task 2.2: Experimental Campaign II

Task 2.3: Baseline Engine Simulation

Task 3.1: Knock Model Validation with Experimental Data

In the project, a new combustion modeling framework for engine knock prediction has been developed. In the developed modeling framework, end-gas ignition is modeled using the conditional moment closure (CMC) [1,2], while spark-ignited premixed flame propagation is modeled using the front propagation formulation (FPF) [3]. Turbulence-chemistry interactions during end-gas ignition are described in CMC by adopting filtering over iso-enthalpy surfaces, which preserves 
small-scale scalar structures to a certain degree and helps to estimate chemical reaction rates with consideration of turbulence-chemistry interactions. The weak spatial dependence of the surfacefiltered quantities (or quantities filtered with the condition that enthalpy has a certain value) allows for the use of a coarse CMC grid in the spatial coordinates - details of scalar structures and turbulence-chemistry interactions are described in enthalpy space. As a result, assuming the weak spatial dependence, the method allows for the use of a reasonably detailed chemical mechanism for end-gas ignition in LES with a reasonable computational cost. Being developed as a method to minimize numerical artifacts in simulating the propagation of filtered premixed flame fronts, which are usually thin and under-resolved in LES, FPF is combined with CMC and extended for use in the engine knock simulation. The model formulation that consistently combines FPF with CMC has been developed.

The developed modeling framework, CMC-FPF, has been implemented as the user defined function (UDF) of CONVERGE CFD and validated for engine experiments. As the conditioning variable used in CMC, which is used for surface filtering, total enthalpy and sensible enthalpy are adopted. Although the analysis of DNS data for end-gas ignition, which was generated during this project, suggests that the sensible enthalpy conditioning works better in terms of estimating the filtered chemical reaction rates during end-gas ignition, the validated version of the CMCFPF code is based on total enthalpy conditioning. The sensible enthalpy version is implemented but is not fully tested. One reason behind it is that according to a numerical test for a simple configuration (2-D DNS of end-gas ignition in homogeneous turbulence), the difference between the two methods in terms of predicting ignition delay appears to be much smaller than observed in a priori analysis of the DNS data in terms of estimating the filtered reaction rates. This may be because thermal runaway in the stoichiometric mixture occurs over a very short time period. The current version of the CMC-FPF UDF code is based on the spatially-integrated form of the $\mathrm{CMC}$ equation, where the spatial dependence of the surface-filtered quantities is neglected. This formulation is expected to work well when end-gas ignition occurs in a single spot that maintains a high temperature favorable for ignition. The validation has been performed for the stoichiometric operations with and without the exhaust gas recirculation (EGR). The fuel tested is iso-octane. The in-cylinder pressure measurements in a single-cylinder version of the GM LNF 2.0L 4-cylinder design turbocharged gasoline direct injected (GDI) engine from the 2007 Pontiac Solstice were used for validation. The gas sampling measurements, which were originally planned, were not made. The results show that the developed CMC-FPF method captures knocking in stoichiometric operation cases studied. However, it did not capture knocking in a case with EGR.

Besides the tasks that were originally planned, a laminar-to-turbulent flame transition model has been developed to capture cycle-to-cycle variations (CCVs) in the burning rates in a spark ignition (SI) engine, which affect the thermodynamic trajectory of the end gas and thus its ignition delay. The results show that, at least, for conditions studied in this project, the modeling of variations in the early-stage burning rate is critical to capturing the degree of CCVs in incylinder pressure. The developed transition model captures the effects of turbulence intensity on the laminar-to-turbulent flame transition, based on the Kolmogorov similarity hypothesis, and can be used in other premixed combustion models. The analysis of the factors affecting CCVs in the engine experiment was performed.

In what follows, the model formulation and the development of the computational code used in this project are described (Tasks 1.1, 2.3 and 3.1). In Sec. 3.1, the representative accomplishments for DNS of end-gas ignition and its use in knock model development are described (Tasks 1.2 and 
2.1). In Sec. 3.2, the accomplishments in the experimental campaigns are described (Tasks 1.2 and 2.2). In Sec. 3.2, the validation for a non-knocking case is presented with emphasis on capturing the degree of CCVs (Task 2.3). In Sec.3.4, the validation for knocking cases is presented (Task 3.1). The products resulted from the project are summarized at the end.

\section{Combustion Model for Knock Prediction}

In this section, the developed combustion model, CMC-FPF, and the solver are described. In the developed modeling framework, end-gas ignition processes are described by the CMC model, while the propagation of spark-ignited premixed flames is described by FPF. The model is implemented as user defined functions (UDF) for CONVERGE CFD.

\subsection{CMC-FPF Model}

The CMC and FPF models are combined using the Heaviside function that is unity in the end-gas region and zero otherwise. The Heaviside function or the end-gas indicator is determined by the premixed-flame progress variable, $c_{p}$, which is solved in the FPF model. Specifically, conditionally filtered scalar quantities that are used to describe end-gas ignition are defined as

$$
Q_{i}(\eta ; \mathbf{x}, t)=\int_{V} \rho Y_{i} \delta(h-\eta) H\left(c_{p}^{*}-c_{p}\right) F(\mathbf{x}) d V / \int_{V} \rho \delta(h-\eta) H\left(c_{p}^{*}-c_{p}\right) F(\mathbf{x}) d V,
$$

where $H$ is the Heaviside function that has a non-trivial value (unity) only in the end-gas region and, therefore, by weighting the sampling variable (species mass fraction in the above equation) with the Heaviside function, only the end-gas region is taken into account in obtaining the filtered (averaged) species mass fraction. Being weighted by the Dirac delta function, the sample is taken only when the mixture has a particular value of the enthalpy $h$. Because the enthalpy in homogeneous mixture composition highly correlates with temperature, which strongly affects ignition chemistry of end-gas, the end-gas ignition can be well predicted by using the conditionally filtered species mass fractions. $\rho$ is the density. $F$ is the spatial filtering that is associated with a particular CMC cell and extends from a flame surface to a cylinder wall. In the above equation, scalar structures within the physical domain where $F$ is significant is mapped onto scalar (enthalpy) space, $\eta$.

The equations for end-gas ignition $\mathrm{CMC}$ can be written as

$$
\frac{\partial Q_{i}}{\partial t}+\overline{\mathbf{v} \mid \zeta} \cdot \nabla Q_{i}+T_{Y_{i}^{\prime}}=-v_{\eta} \frac{\partial Q_{i}}{\partial \eta}+\left\langle N_{\xi} \mid \eta\right\rangle \frac{\partial^{2} Q_{i}}{\partial \eta^{2}}+\frac{\left\langle\rho \omega_{i} \mid \eta\right\rangle}{\rho_{\eta}}
$$

where $Q_{i}$ is the conditional mean mass fraction of species $i . \mathbf{v}$ is the fluid velocity. $v_{\eta}$ is the convective velocity in $\eta$ space, which results from a source term in the equation for a conditioning variable. $N_{\xi}$ is the scalar dissipation rate defined as $N_{\xi}=D \nabla \xi \cdot \nabla \xi$, where $D$ is the molecular diffusivity and $\xi$ is the normalized enthalpy. The unity Lewis number is assumed. $\omega_{i}$ is the chemical reaction rate of species $i$. It represents the rate of small-scale mixing processes, which affect chemical reaction rates. The term $T_{Y_{i}^{\prime}}$ represents the effects of the conditional sub-filter fluctuations and can be modeled using the gradient transport assumption. The filtered chemical reaction rates are approximated as

$$
\left\langle\rho \omega_{i} \mid \eta\right\rangle=\rho_{\eta} \omega_{i}\left(\rho_{\eta}, \mathbf{Q} ; \eta\right)
$$


where the conditionally-filtered species mass fractions $\mathrm{Q}$ are directly used to approximate the conditionally-filtered reaction rates. This first-order closure of chemical reaction rates is expected to work well when conditional filtering (or surface filtering) suppresses variations of scalars within a filter by preserving small-scale scalar structures. Once the conditionally filtered species mass fractions are determined in $\mathrm{CMC}$, the filtered species mass fractions, which are needed in a flow solver, can be obtained by the integration over enthalpy space:

$$
\tilde{Y}_{i}=\int_{0}^{1} Q_{i} \widetilde{P}(\eta) d \eta
$$

where $\widetilde{P}(\eta)$ is the density-weighted filtered density function (FDF) of the conditioning variable. The conditioning variable, enthalpy, is normalized to have a value between 0 and 1 . The FDF is obtained by assuming its shape, which is parameterized by the filtered mean and subfilter variance of the conditioning variable.

The following (density-weighted) filtered progress variable equation is solved in FPF:

$$
\frac{\partial \bar{\rho} \widetilde{c_{p}}}{\partial t}+\nabla \cdot\left(\bar{\rho} \widetilde{\mathbf{v}} \widetilde{c_{p}}\right)=-\nabla \cdot\left(\overline{\rho \mathbf{v} c_{p}}-\bar{\rho} \widetilde{\mathbf{v}} \widetilde{c_{p}}\right)+\nabla \cdot\left(\overline{\rho D \nabla c_{p}}\right)+\bar{\rho} \widetilde{\omega}_{c, p}+\bar{\omega}_{s p k},
$$

where $c_{p}$ is the reaction progress variable due to spark-ignited premixed flame propagation, and $\omega_{c, p}$ is the chemical reaction term for the progress variable. The overline and the tilde denote filtering and density-weighted filtering, respectively. $\omega_{s p k}$ is the source term due to spark ignition. In FPF, the filtered reaction rates are written as [3]

$$
\widetilde{\omega}_{c, p}=\frac{\rho_{u}}{\bar{\rho}} s_{t} \delta_{\Delta}\left[\mathbf{n} \cdot\left(\mathbf{x}-\mathbf{x}_{s}\right)\right]
$$

where $s_{t}$ is the propagation speed of the filtered flame front, $\rho_{u}$ the density of the unburned mixture, and $\delta_{\Delta}\left[\mathbf{n} \cdot\left(\mathbf{x}-\mathbf{x}_{s}\right)\right]$ the regularized delta function. Since the integration of the regularized delta function from the unburned to the fully buned side along the flame normal direction leads to unity, the formulation preserves the total reaction rates and thus minimizes the spurious propagation of the filtered flame front on an LES grid. With the regularized delta function being modeled as $\left|\nabla \psi\left(\widetilde{c_{p}}\right)\right|$, the filtered reaction rates can be written as

$$
\widetilde{\omega}_{c, p}=\frac{\rho_{u}}{\bar{\rho}} s_{t}\left|\nabla \psi\left(\widetilde{c_{p}}\right)\right|,
$$

where $\psi$ is the front structure function [3]. It is modeled as the second-order polynomial of $\widetilde{c_{p}}$.

\subsection{CMC-FPF Solver}

For use in engine LES, CMC-FPF is implemented into CONVERGE CFD as UDF.

\subsubsection{CMC Solver}

The base CMC solver for end-gas ignition has been developed by extending the CMC solver developed for non-premixed combustion $[4,5]$. In the version implemented for coupling with CONVERGE CFD, the spatially-averaged form of the CMC equation is solved, which can be written as

$$
\frac{\partial Q_{i}^{e}}{\partial t}=-v_{\eta} \frac{\partial Q_{i}^{e}}{\partial \eta}+\left\langle N_{\xi} \mid \eta\right\rangle^{e} \frac{\partial^{2} Q_{i}^{e}}{\partial \eta^{2}}+\frac{\left\langle\rho \dot{\omega}_{i} \mid \eta\right\rangle^{e}}{\rho_{\eta}}
$$


$Q_{i}^{e}$ is the average of $Q_{i}$ over the entire end-gas region in the cylinder.

Equation (8) is solved by using the stiff-ODE solver, VODE [6]. The second-order central finite difference scheme is used to discretize the diffusion term in $\eta$ space, the second term on the r.h.s. A semi-Lagrangian-Eulerian treatment is used to deal with the convective term, the first term on the r.h.s. The CHEMKIN II package is used for evaluating chemical reaction rates and thermodynamic/transport properties.

\subsubsection{Conditioning Variable in CMC}

For ignition problems, enthalpy is a good candidate for a conditioning variable used in CMC. In a spark ignition (SI) engine where the stratification of the mixture composition is weak, temperature is expected to have the greatest effect on ignition delay. Due to strong nonlinear dependence of combustion reaction rates on temperature, temperature fluctuations are closely linked to fluctuations of the reaction rates in a turbulent flow inside the cylinder. As a result, when filtering is taken on an iso-enthalpy surface (filtering conditioned with enthalpy), temperature fluctuations within the filter are suppressed and chemical reaction rates can be estimated accurately using the conditionally filtered scalars. Total enthalpy, which includes both chemical and thermal components, or sensible enthalpy, which includes only the thermal component, can be used as the conditioning variable. To deal with a knock problem, temperature fluctuations associated with the propagation of ignition-induced pressure waves have to be removed from those in the conditioning variable. The low Mach number enthalpy, defined below, is proposed to meet such a need.

\section{Pseudo Enthalpy: Separating Pressure Wave Induced Enthalpy Fluctuations}

When knock happens, strong pressure waves are generated and propagate in the cylinder. Such pressure waves are accompanied with local fluctuations of enthalpy. In order to consider the effects of temperature fluctuations on end-gas ignition properly in CMC, enthalpy fluctuations induced by the pressure wave propagation are to be separated from those due to thermal stratification, originated from various other sources including variations in wall temperature along the engine components. Otherwise, the pressure-wave-induced fluctuations in enthalpy introduce unphysical oscillations in filtered species mass fractions since the filtered species mass fractions are determined by the FDF of the conditioning variable, enthalpy. To remedy this problem, it is proposed that the low Mach number assumption is applied to the equation for enthalpy. In other words, instead of enthalpy in a real compressible flow, the pseudo-enthalpy that separates pressure-wave-induced enthalpy fluctuations from thermal-stratification-related fluctuations is used as a conditioning variable in CMC. For that purpose, in addition to an energy equation solved in a flow solver, another equation for the pseudo-enthalpy, where enthalpy fluctuations due to pressure waves are removed, is to be solved. For total enthalpy, the pseudo-enthalpy equation reads

$$
\frac{\partial \bar{\rho} \widetilde{h}_{t}}{\partial t}+\frac{\partial \bar{\rho} \widetilde{h_{t}} \tilde{u}_{i}}{\partial x_{i}}=\frac{\partial \bar{p}}{\partial t}+\frac{\partial}{\partial x_{i}}\left[\bar{\rho}\left(\widetilde{D}+D_{t}\right) \frac{\partial \tilde{h}_{t}}{\partial x_{i}}\right],
$$

where $h_{t}$ is the pseudo enthalpy, $\bar{p}$ is the cylinder-averaged thermodynamic pressure, $D$ is the molecular diffusivity, $D_{T}$ is the subfilter turbulent diffusivity, $u_{j}$ and $x_{j}$ are the velocity component and the space coordinate in the direction $j$. The tilde denotes the density-weighted filtering. The above equation is equivalent to the enthalpy equation under the low Mach number assumption. By using the cylinder-averaged thermodynamic pressure, the pressure-wave-induced enthalpy 
flctuations are removed. The underlying assumption behind the use of the pseud enthalpy is that fluctuations in thermochemical variables caused by the passage of pressure waves do not affect chemical reaction rates significantly.

\section{Mixture Stratification and Enthalpy Correction}

For SI engines with direct injection (DI), different levels of mixture stratification may exist depending on the mixing of evaporated fuel and air. In a GDI engine operated with early injection, the level of the mixture composition stratification is relatively weak. In the stratified case, enthalpy of the mixture depends not only on temperature but also on the mixture composition and the equivalence ratio. Since we assume the homogeneous mixture composition in CMC, this dependence of enthalpy on the mixture composition has to be compensated, although the level of stratification is weak, in order to consider thermal fluctuations correctly.

The purpose of the compensation is to generate an enthalpy field that is consistent with actual thermal stratification. To reproduce thermal stratification correctly, enthalpy of a mixture with the local equivalence ratio $\widetilde{\phi}$ is changed to enthalpy of a mixture with the global equivalence ratio $\bar{\phi}$, which is the cylinder-averaged equivalence ratio. During the change, temperature remains the same. Thus, the dependence of enthalpy on the mixture composition fluctuations is removed, while thermal stratification is preserved. The correction is made based on $h_{t}-\phi$ curve for a given value of the temperature $\widetilde{T}$ as,

$$
\hat{h}_{t}=\widetilde{h}_{t}+\frac{\Delta h_{t}}{\Delta \phi}(\widetilde{\phi}-\bar{\phi})
$$

where $\hat{h}_{t}$ is the corrected-enthalpy, and $\widetilde{h_{t}}$ is the uncorrected enthalpy. $\frac{\Delta h_{t}}{\Delta \phi}$ is the slope of the $h_{t}-\phi$ relation at given temperature $\widetilde{T}$.

Alternatively, before heat release due to end-gas ignition becomes significant, the mixture enthalpy can be calculated, at each LES grid point, using the local temperature and the cylinderaveraged species mass fractions. The resulting field is set as the initial field for the pseudo-enthalpy, which becomes the conditioning variable from that time on.

\subsubsection{Coupling with CONVERGE CFD}

CONVERGE solves for species mass fractions and internal energy to evaluate density through which coupling of the scalars with pressure and velocity is made in the PISO (Pressure Implicit with Operator Splitting) loop. On the other hand, CMC simulates ignition using a reduced chemical mechanism consisting of hundreds species. Since the information on the ignition process is contained in the CMC part, only the information needed to calculate the density and the heat release is necessary on the CONVERGE side. For this purpose, solving a reduced set of species, e.g., major species only, on CONVERGE is sufficient. The benefit is the reduction of computational cost. An approach to enforce the CMC solution into the CONVERGE solution through coupling using a reduced set of species is developed.

In the CONVERGE UDF, the equations for the filtered species mass fractions are solved using 
an operator splitting method [7]:

$$
\begin{aligned}
\frac{\bar{\rho}^{n}\left(\widetilde{Y}_{i}^{n+1, *}-\widetilde{Y}_{i}^{n}\right)}{\Delta t} & =\overline{\rho \omega_{i}} \\
\frac{\left(\bar{\rho} \widetilde{Y}_{i}\right)^{n+1}-\left(\bar{\rho} \widetilde{Y}_{i}\right)^{n+1, *}}{\Delta t} & =\frac{\delta}{\delta x_{j}}\left[\bar{\rho}\left(D+D_{T}\right) \frac{\delta \widetilde{Y}_{i}}{\delta x_{j}}\right]-\frac{\delta \bar{\rho} \widetilde{u}_{j} \widetilde{Y}_{i}}{\delta x_{j}},
\end{aligned}
$$

where $\bar{\rho}$ is the filtered density, $\widetilde{Y}_{i}$ is the filtered mass fraction of species $i$, and $\overline{\rho \omega_{i}}$ is the filtered chemical reaction rate. The superscripts $n$ and $n+1$ denote the values at the current and the next time step, respectively, and the superscript $(n+1, *)$ denotes the value after the chemical reaction step. $\delta / \delta x_{j}$ denotes a discrete spatial derivative operator. In the simulations performed in this project, only seven major species - fuel, $\mathrm{O}_{2}, \mathrm{~N}_{2}, \mathrm{CO}_{2}, \mathrm{CO}, \mathrm{H}_{2} \mathrm{O}$, and $\mathrm{H}_{2}-$ are solved. The CMC solution is enforced by properly evaluating the filtered chemical reaction term, $\bar{\rho} \omega_{i}$, in the equations for those species. At the chemical reaction step, the conditionally filtered reacting scalars are solved in CMC and the CMC solutions are translated to the filtered reacting scalars used in the flow solver through a coupling method described below. Then, at the transport step, the transport operators are solved in the PISO algorithm used in CONVERGE.

The filtered species mass fraction after the chemical reaction step of the operator splitting method, $\widetilde{Y}_{i}^{n+1, *}$, is evaluated as

$$
\tilde{Y}_{i}^{n+1, *}=\tilde{Y}_{i}^{n+1, A *} \cdot w+\tilde{Y}_{i}^{n+1, B *} \cdot(1.0-w),
$$

where $\widetilde{Y}_{i}^{n+1, A *}$ and $\widetilde{Y}_{i}^{n+1, B *}$ are given as

$$
\begin{gathered}
\widetilde{Y}_{i}^{n+1, A *}=\int_{0}^{1} Q_{i}^{n+1} \widetilde{P}^{n}(\eta) d \eta \\
\widetilde{Y}_{i}^{n+1, B *}=\widetilde{Y}_{i}^{n}+\int_{0}^{1}\left(Q_{i}^{n+1}-Q_{i}^{n}\right) \widetilde{P}^{n}(\eta) d \eta .
\end{gathered}
$$

$\widetilde{P}(\eta)$ is the FDF of the conditioning variable or normalized enthalpy. In Eq. (14), the filtered species mass fraction follows the solution in CMC which assumes perfectly-mixed fuel/air composition. Since the CMC solutions are imposed to the filtered scalar fields, Eq. (14) cannot capture the mixture composition stratification in a GDI engine. On the other hand, Eq. (15) preserves the mixture composition stratification, which is weak for the conditions of interest in this project. It is thus a preferable coupling method when the mixture stratification is non-negligible. However, it is found that Eq. (15) can lead to numerical instability when heat release rates are high. Here, to overcome such shortcomings in each coupling method, a hybrid approach where the coupling method gradually changes from Eq. (15) to Eq. (14) is used. $w$ is a weighting factor representing the transition. $w$ is 0 initially. Equation (15), which preserves the mixture composition stratification, is thus used initially when the mixture stratification is not negligible. $w$ increases gradually to 1.0 as heat release rates increase. $w$ is then modeled as

$$
w= \begin{cases}0, & t<t_{0} \\ \frac{t}{\tau_{h}}, & t_{0}<t<t_{0}+\tau_{h} \\ 1.0, & t>\tau_{h}\end{cases}
$$


where $t_{0}$ is the time at which the transition between the coupling methods starts, and $\tau_{h}$ is a time duration for which the transition occurs.

Since the chemical source terms in the equations of the major species solved in CONVERGE are determined to follow the CMC solution, the sum of the mass fractions of the major species is less than unity. An approach to compensate this deficit mass fraction, defined below in Eq. (17), is necessary to conserve mass in CONVERGE:

$$
\widetilde{Y}_{d}=1-\sum_{k}^{N_{m}} \int_{0}^{1} Q_{k} \widetilde{P}(\eta) d \eta
$$

where $\widetilde{Y}_{d}$ is the deficit mass fraction that has to be redistributed, and $N_{m}$ represents the number of species solved for in CONVERGE. The deficit mass fraction can be distributed into fuel, oxygen and final combustion products $\left(\mathrm{C}_{8} \mathrm{H}_{18}, \mathrm{O}_{2}, \mathrm{CO}_{2}\right.$, and $\left.\mathrm{H}_{2} \mathrm{O}\right)$ according to the progress of overall reactions, while satisfying element conservation. An investigation has shown that depositing the deficit mass using a progress variable based on heat release works well:

$$
c_{h}=\frac{\text { heat release in CMC }}{\text { heat released when burned completely }}=\frac{-\sum_{i}^{N} \int_{0}^{1} Q_{i} P(\eta) d \eta h_{f, i}+\sum_{i}^{N} \widetilde{Y}_{i}^{u} h_{f, i}}{-\sum_{i}^{N} \widetilde{Y}_{i}^{b} h_{\dot{f}, i}+\sum_{i}^{N} \tilde{Y}_{i}^{u} h_{\dot{f}, i}} \text {, }
$$

where the summation is done over the whole species set considered in CMC, and $N$ represents the number of species in the whole set. $\widetilde{Y}_{i}^{u}$ is the filtered species mass fraction of species $i$, calculated assuming that the mixture is unburned. $\widetilde{Y}_{i}^{b}$ is the filtered mass fraction of species $i$, calculated assuming that the mixture is completely burned. $h_{f, i}$ is the enthalpy of formation. The deficit mass is distributed into the reactants and the products according to the progress variable $c_{h}$, so that heat release in CONVERGE matches with that in CMC.

A similar approach is used for coupling of FPF with CONVERGE. The mixture composition is described using seven major species: fuel, $\mathrm{O}_{2}, \mathrm{~N}_{2}, \mathrm{CO}_{2}, \mathrm{CO}, \mathrm{H}_{2} \mathrm{O}$, and $\mathrm{H}_{2}$. The following equation for the density-weighted filtered species mass fraction, $\widetilde{Y}_{i}$, is solved:

$$
\frac{\partial \bar{\rho} \widetilde{Y}_{i}}{\partial t}+\frac{\partial \bar{\rho} \widetilde{u}_{j} \widetilde{Y}_{i}}{\partial x_{j}}=\frac{\partial}{\partial x_{j}}\left(\bar{\rho}\left(\widetilde{D}+D_{t}\right) \frac{\partial \widetilde{Y}_{i}}{\partial x_{j}}\right)+\bar{\rho} \widetilde{\omega}_{i}
$$

Using the source term for the progress variable, the source term for the species mass fraction, $\bar{\rho} \widetilde{\omega}_{i}$, is calculated as,

$$
\bar{\rho} \widetilde{\omega}_{i}=\bar{\rho} \widetilde{\omega}_{c, p}\left(Y_{i}^{b}-Y_{i}^{u}\right),
$$

where $Y_{i}^{b}$ and $Y_{i}^{u}$ represent the local burned and the unburned mass fraction of species $i$, respectively. The composition of the burned mixture is calculated using the CEQ library [8] by assuming the burned mixture is at chemical equilibrium. The unburned mass fractions $Y_{i}^{u}$ are obtained by solving a separate set of species transport equations which have no chemical source term.

The CMC equations are solved from well before spark discharge till all the mixture being burned. For instance, in one of the simulations described in the validation part, the ignition simulation by CMC started at 100 crank angle degrees (CAD) before the top dead center (TDC). At the start of solving the CMC equations, the species mass fractions in the cylinder are averaged to provide the initial condition for CMC. This allows for considering the residual gas in CMC. The 
mixtures with the equivalence ratio $\phi$ being close to 1 , e.g., $0.9<\phi<1.1$, are considered and the very rich and lean mixtures are excluded when averaging. Using the average species mass fractions and the local temperature, enthalpy at each LES grid point is evaluated. The resulting enthalpy field is taken as the initial field for the pseudo enthalpy, which is the conditioning variable. Well before TDC, heat release rates are negligible but to allow the formation of the radical pool, CMC equations are solved. However, the changes in the density and flow fields due to chemical reactions during this period are very small. Thus, in order to reduce the computational cost, the reaction rates in the filtered species mass fractions solved in CONVERGE are neglected when heat release rates and the change in the molecular weight of the mixture due to chemical reactions are negligible. The enthalpy evaluated using the average species mass fractions and the local temperature, as described above, is used as a conditioning variable when the reaction rates in the filtered species mass fractions are negelcted. In one of the simulations described later in the validation part, they are neglected until $30 \mathrm{CAD}$ before TDC. After that, the full coupling between CMC and CONVERGE is done according to the approach described above.

Results: The effects of the species subset size and the deficit mass deposit method on the ignition delay prediction is studied for constant-volume ignition in 2-D homogeneous isotropic turbulence. LES is performed. The computational domain is a square box with the side length of $3 \mathrm{~mm}$. The periodic boundary conditions are used. The uniform iso-octane and air mixture with an equivalence ratio of 1.0 is considered. The velocity field is initialized by the Passot-Pouquet spectrum [9] with $\bar{u}_{0}=0.0 \mathrm{~m} / \mathrm{s}, u_{0}{ }^{\prime}=1.0 \mathrm{~m} / \mathrm{s}$. The temperature field is initialized by the Passot-Pouquet spectrum modified for scalars with $\bar{T}_{0}=800 \mathrm{~K}$ and $\bar{T}_{0}{ }^{\prime}=10 \mathrm{~K}$. The integral length scale for both velocity and temperature fields is $1.2 \mathrm{~mm}$. The dynamic structure model is used for sub-filter turbulence modeling. The reduced chemical mechanism for the primary reference fuel (PRF) $[10,11]$ is used. In CONVERGE, three species subsets are considered: the 7 major species, 13 species (7 major species and other minor species), and all species. Cases with different initial pressures, $20 \mathrm{bar}, 30 \mathrm{bar}, 40 \mathrm{bar}$ and $50 \mathrm{bar}$, are studied. The thermodynamic conditions are relevant to engine conditions near TDC.

Figure 1 shows the pressure evolution. It can be seen that using 7 major species with the fuelmass-fraction-based progress variable, the ignition delays are underpredicted (around 10\%) for the four cases. When 13 species are used in CONVERGE, the ignition delays are still underpredicted by around $4 \%$. This underprediction is because heat release in CONVERGE is larger than that in $\mathrm{CMC}$ when using the fuel-mass-fraction-based progress variable. The use of the heat-releasebased progress variable $c_{h}$ substantially improves the prediction of ignition delays. Even when the 7 major species are solved for in CONVERGE, the prediction is within 1\% error as compared with that using all the species. Based on this, in LES of knock described below, the 7 major species are solved for in CONVERGE, while the heat-release-based progress variable is used to deposit the deficit mass.

\subsubsection{Laminar-to-Turbulent Flame Transition Model: Reproducing Cycle-to-Cycle Varia- tions in SI Premixed Burning}

The burning rates due to SI premixed flame propagation affect the thermodynamic trajectory of end gas and the knocking tendency. It has been known that the premixed burning rates can vary substantially for different cycles. Early investigations have shown the importance of early-stage 

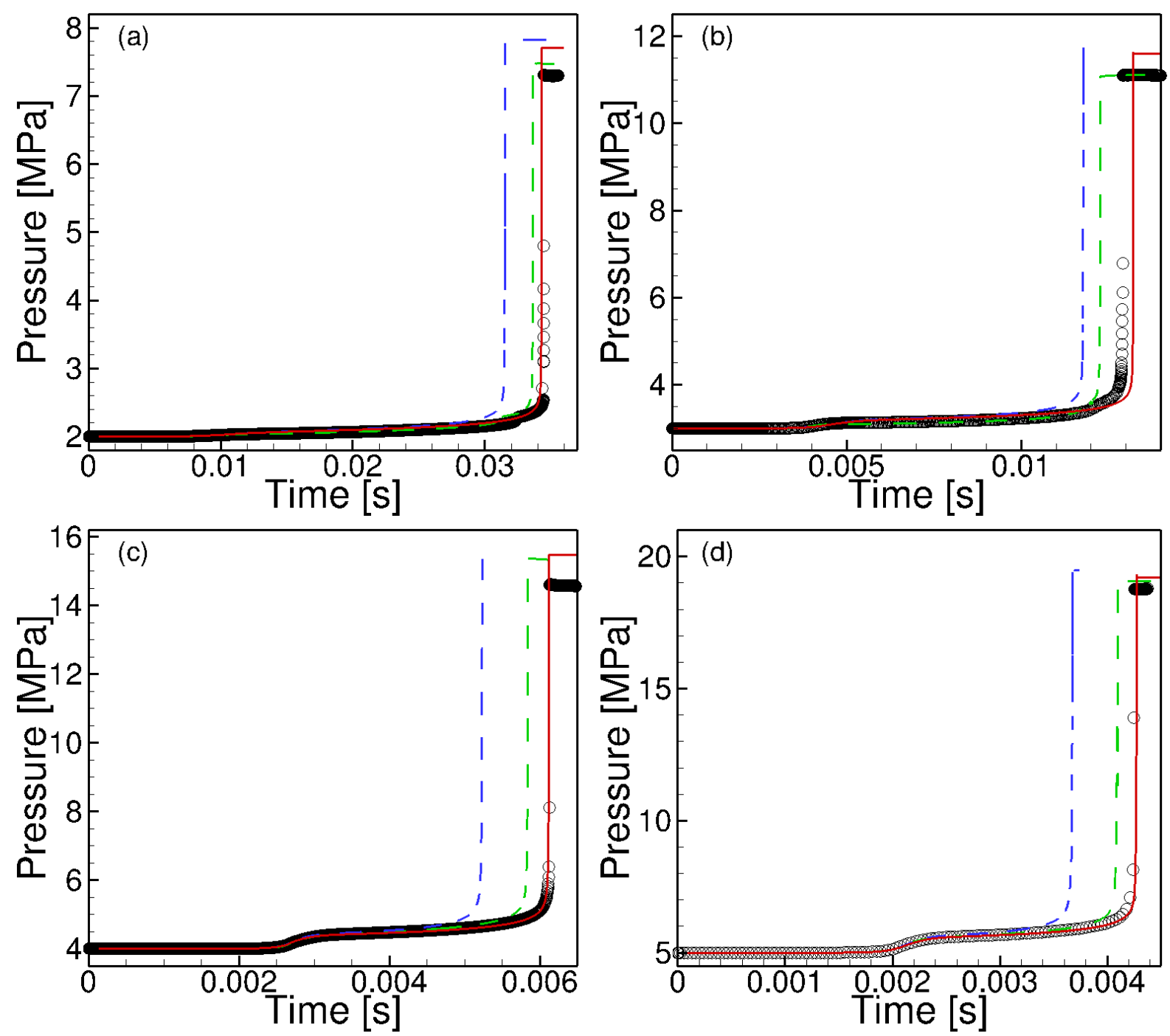

Figure 1: Effects of the species set solved for in CONVERGE and the deficit mass deposition method on ignition delay times in the 2-D constant volume ignition cases. Pressure evolution indicating the ignition delays are shown. (a) $P_{0}=20$ bar. (b) $P_{0}=30$ bar. (c) $P_{0}=40$ bar. (d) $P_{0}=50$ bar (black circles: 121 species, green dashed lines: 13 species with the fuel-based progress variable for deficit mass deposition, blue dashed lines: 7 major species with the fuel-based progress variable for deficit mass deposition, red solid lines: 7 major species with the heat-released-based progress variable for deficit mass deposition).

burning rates in the $\mathrm{CCV}$, which are affected by the laminar-to-turbulent flame transition occurring in the early-stage flame kernel development. Recent experimental studies by Schiffmann et al. [12] and Zeng et al. [13] also suggest the importance of variations in the laminar-to-turbulent flame transition in CCV. As shown in Fig.4(a) of Zeng et al. [13], there are substantial variations in the flame surface area during the early stage of the burning process where the laminar-to-turbulent flame transition occurs. Schiffmann et al. [12] performed a statistical analysis of their PIV and $\mathrm{OH}$ fluorescence data obtained in an optical engine in order to look for a root cause of CCV in an SI engine. Among many quantities, the laminar-to-turbulent flame transition time is found to have strong correlation with CCV (Fig. 13 of Schiffmann et al. [12]). 
In this project, a laminar-to-turbulent flame transition model is developed to capture CCV in an SI engine. In what follows, the transition model as well as a model used to describe the spark ignition process is described.

The spark ignition is described by extending a model of Richard et al. [14]. The flame kernel is generated by depositing the burned gas at the location of the spark plug. The deposited progress variable is given by

$$
\bar{c}_{s p k}=c_{0} \exp \left[-\frac{\left|\mathbf{x}-\mathbf{x}_{s p k}\right|^{2}}{\left(0.6 \Delta_{f}\right)^{2}}\right]
$$

where $\mathbf{x}_{s p k}$ is the location of the spark plug and $\Delta_{f}$ is the characteristic scale, which is set to be close to the filter size near the spark plug in this work. $c_{0}$ can be determined to match the total deposited mass as,

$$
\dot{m}_{s p k}=\frac{4}{3} \pi \rho_{b} r_{s p k}^{0}=\int \rho_{b} \bar{c}_{s p k} d v
$$

where $\dot{m}_{s p k}$ is the total deposited mass, $\rho_{b}$ is the density of the burned gas, and $r_{s p k}^{0}$ denotes the radius of the initially deposited kernel. $r_{s p k}^{0}$ is assumed to be several times of the laminar flame thickness, $\delta_{l} . \bar{c}_{s p k}$ can be deposited through the source term in the filtered progress variable equation as,

$$
\bar{\omega}_{s p k}=\frac{\rho_{b} \bar{c}_{s p k}-\bar{\rho} \tilde{c}}{t_{s p k}}
$$

where $t_{s p k}$ is the spark characteristic time and can be simply set as the time step. After the initial deposition, the reaction source term for the progress variable is modeled as,

$$
\bar{\omega}_{s p k}=\rho_{u} \alpha \widetilde{c}_{p}\left(1-\widetilde{c}_{p}\right)
$$

where $\alpha$ is calculated as,

$$
\alpha=\frac{4 \pi r_{f}^{2}\left\langle s_{t}\right\rangle_{s}}{\int \widetilde{c}_{p}\left(1-\widetilde{c}_{p}\right) d v} .
$$

$\langle\cdot\rangle_{s}$ denotes averaging over the filtered flame surfaces. The spark ignition model is used until the maximum progress variable in the domain reaches one. After that, the progress variable source term is evaluated using the FPF method.

During the early development of the flame kernel, it is assumed that the propagation speed of the filtered flame front $s_{t}$ gradually increases from the laminar flame speed, $s_{L}$, to the equilibrium state turbulent flame speed, $s_{t}^{e q}$. This process is modeled as,

$$
s_{t}=(1-w) s_{L}+w s_{t}^{e q} .
$$

$w$ is the weight controlling the transition from the laminar flame to the fully turbulent flame where the production and destruction of flame surfaces within a filter scale are balanced. The dependence of the weight $\omega$ on the flame kernel radius $r_{f}$ is modeled as

$$
w=\frac{1}{2}\left\{\tanh \left[\frac{4\left(r_{f}-\eta_{i}\right)}{\eta_{o}-\eta_{i}}-2\right]+1\right\},
$$

where $\eta_{i}$ represents the flame radius where the laminar-turbulent flame transition starts and $\eta_{o}$ the flame radius where the kernel becomes fully turbulent. $\eta_{i}$ and $\eta_{o}$ are called the inner transition scale and the outer transition scale, respectively, here. 


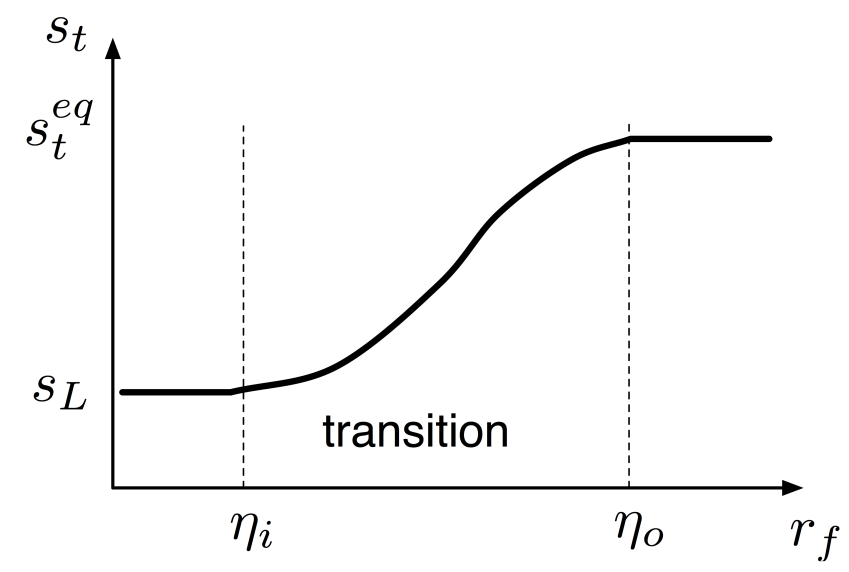

Figure 2: Illustration of the laminar-to-turbulent flame transition model.

In the present model, variations in the laminar-to-turbulent flame transition time are considered through variations in the outer transition scale at which a flame kernel becomes fully turbulent. The laminar-to-turbulent flame transition is controlled by the interaction between flame propagation and turbulence. The more energetic is turbulence, the sooner will the kernel transit from the laminar to the turbulent flame. The inner scale, $\eta_{i}$, is expected to be less sensitive to this interaction and can thus be approximated as a constant on the order of the laminar flame thickness, $l_{F}$. Variations in the outer transition scale, $\eta_{o}$, due to varying turbulence intensity is expected to account for the major portion of variations in the laminar-to-turbulent flame transition time.

The Gibson scale represents the size of the smallest eddies that can wrinkle a flame [15]. It can thus be hypothesized that a flame becomes fully turbulent when the size (radius) of the flame kernel becomes a multiple of the Gibson scale. This leads to

$$
\eta_{o}=k l_{G}+\eta_{c}
$$

where $k$ and $\eta_{c}$ are the model parameters. It is assumed that the outer scale is linearly proportional to the Gibson scale and the proportionality constant $k$ is larger than 1 . The cut-off scale $\eta_{c}$ implicitly incorporates the time delay [16] from the spark discharge to the start of the flame transition, which can be assumed to be independent of the turbulence field. It is also used to relax the effects that variations in the energy cascade near a wall have on the evaluation of the Gibson scale.

Adopting the Kolmogorov similarity hypothesis, which assumes that the kinetic energy is transferred from large to small scales without production or dissipation over a certain range of length scales and thus the energy transfer rate is invariant of the length scale in the range, the so-called inertial subrange, the Gibson scale $l_{G}$ can be estimated as

$$
l_{G}(t)=\frac{\left\langle s_{L}\right\rangle^{3}}{\left\langle\epsilon_{\Delta}\right\rangle},
$$

where $s_{L}$ is the (unstretched) laminar flame speed, $t$ time, and $\epsilon_{\Delta}$ the dissipation rate. Assuming the filter scale is in the inertial subrange, the dissipation rate is evaluated as $\left(u_{\Delta}^{\prime}\right)^{3} / \Delta$, where $u_{\Delta}^{\prime}$ is the turbulence velocity scale at the filter size $\Delta$. The angular brackets, $\langle\cdot\rangle$, represent surface averaging. The dissipation rate and the laminar flame speed are averaged over the filtered flame 
surface according to

$$
\left\langle\epsilon_{\Delta}\right\rangle=\frac{\int_{V}\left(\epsilon_{\Delta}\right)_{u}\left|\nabla \widetilde{c}_{p}\right| d v}{\int_{V}\left|\nabla \widetilde{c}_{p}\right| d v}, \quad\left\langle s_{L}\right\rangle=\frac{\int_{V} s_{L}\left|\nabla \widetilde{c}_{p}\right| d v}{\int_{V}\left|\nabla \widetilde{c}_{p}\right| d v},
$$

where $V$ represents the entire computational domain and $\widetilde{c}$ the Favre-filtered progress variable. The subscript $u$ denotes the unburned side. $\left(\epsilon_{\Delta}\right)_{u}$ is evaluated on the unburned side of a filtered flame and then extrapolated across a flame front along a flame normal direction. In the present model, the outer transition scale $\eta_{o}$ scales as the cube of the ratio of $s_{L}$ to $u_{\Delta}^{\prime}$. Thus, the model predicts that higher level of turbulence makes a kernel develop into a fully turbulent flame earlier, which is consistent with the physical picture of the laminar-to-turbulent flame transition.

As the flame kernel grows and moves in a turbulent flow, turbulence near the flame kernel changes. As a simple approach to consider the history effects and smooth out variations of the Gibson scale over time, the temporal-averaged Gibson scale is used to estimate $\eta_{o}$ :

$$
l_{G}=\frac{\int_{t_{0}}^{t} l_{G}(\tau) d \tau}{t-t_{0}}
$$

where $t_{0}$ denotes the time when the spark is discharged.

\section{Accomplishments: Research Activities}

\subsection{Validation Using Direct Numerical Simulation Data}

DNSs have been performed to generate the statistics of several key quantities that appear in the knock model, hence providing the database for model validation and development. 2-D DNSs of ignition in homogeneous isotropic turbulence as well as DNSs for premixed flame propagation have been performed. The suitability of a conditioning variable is investigated. The mixing model for PDF and scalar dissipation of sensible enthalpy is validated. Representative results are presented in this section.

\subsubsection{2-D Reduced Chemistry End-Gas Ignition DNS: Reaction Rate Estimation}

Several DNS cases have been completed for different initial pressures, temperatures and temperature inhomogeneity levels (r.m.s. fluctuations). The fuel/air mixture used is the stoichiometric and lean PRF/air mixtures. The focus of the analysis is on assessing the reaction rate closure in CMC, which can be greatly affected by the choice of a conditioning variable. The CMC model with total enthalpy as the conditioning variable (CMCht) and with sensible enthalpy as the conditioning variable (CMChs) are assessed to find the optimal conditioning variable.

The conditions for representative cases are shown in Table 1. The initial turbulence and temperature fields are generated using the Passot-Pouque spectrum. The linear forcing is used for maintaining turbulence intensity and the temperature inhomogeneity, until ignition occurs. The initial turbulence intensity $u^{\prime}$ is about $1 \mathrm{~m} / \mathrm{s}$, while the initial integral length scale is about $1.2 \mathrm{~mm}$. The governing equations are solved by a second-order finite difference low Mach number variable density solver [17], neglecting the effects of the pressure waves generated during the ignition process and focusing the analysis on the onset of the ignition. A stiff ODE solver, VODE [6], is used 


\begin{tabular}{llllllll}
\hline \hline Case & fuel & $\phi$ & $\bar{T}_{0}[\mathrm{~K}]$ & $T^{\prime}[\mathrm{K}]$ & $P_{0}[\mathrm{bar}]$ & Domain & Grid \\
\hline Case I & PRF80 & 0.7 & 850 & 40 & 30 & $5 \mathrm{~mm}^{2}$ & $3072^{2}$ \\
Case II & PRF80 & 1.0 & 850 & 40 & 30 & $3 \mathrm{~mm}^{2}$ & $2048^{2}$ \\
Case III & PRF80 & 1.0 & 850 & 20 & 30 & $5 \mathrm{~mm}^{2}$ & $3072^{2}$ \\
Case IV & PRF80 & 1.0 & 900 & 20 & 30 & $5 \mathrm{~mm}^{2}$ & $3072^{2}$ \\
\hline \hline
\end{tabular}

Table 1: Simulation conditions for 2-D DNS of PRF80/air end-gas ignition. $\bar{T}_{0}$ is the initial mean temperature. $T^{\prime}$ is the temperature inhomogeneity level or the r.m.s. fluctuation of temperature. $P_{0}$ is the initial pressure.

to time-integrate chemical reaction terms. CHEMKIN II [18] is used to evaluate chemical reaction rates and transport properties. A reduced mechanism for the PRF/air mixture [10,11] is used. The temperature and $\mathrm{H}_{2} \mathrm{O}_{2}$ fields for Case IV are shown in Fig. 3.

\section{$\underline{\text { Results }}$}

In Figs. 4-7, heat release rates predicted by CMCht and by CMChs are compared with the DNS data at four different time instances for Case IV, from the very early stage of ignition processes to the time when the heat release rate reaches its maximum value.

Figure 4 shows the heat release rate prediction at $t=2.5 \mathrm{~ms}$, when heat release rates start increasing but the pressure rise is negligible. Both CMCht and CMChs perform well at this very early ignition stage. It is also clear that the DNS data of heat release rates (black circle) for CMCht are more scattered than for CMChs, suggesting the sensible enthalpy is a better conditioning variable.

Figure 5 shows the heat release rate prediction at $t=2.56 \mathrm{~ms}$. At this time instant, ignition is at an early stage and occurs at some local hot spots. CMChs captures the right physics, while CMCht overpredicts heat release rates. The error in CMCht is due to large fluctuations of temperature and species mass fractions, which originate from the coexistence of unburned, burned, and ignition fronts within the samples having similar total enthalpy values.

Figure 6 shows the heat release rate prediction at $t=2.64 \mathrm{~ms}$. At this time instant, ignition is still at an early stage, with the total heat release rate being less than $10 \%$ of the maximum heat release rate. CMChs still performs well. However, CMCht leads to substantial errors.

Figure 7 shows the heat release rate prediction at $t=2.84 \mathrm{~ms}$, when the total heat release rate is very close to its maximum. CMChs gives very good prediction for heat release rates. The error in CMCht is reduced as compared with that at $t=2.56 \mathrm{~ms}$ and $t=2.64 \mathrm{~ms}$, but still substantial.

The capability of the models in predicting reaction rates of key radicals and species is also assessed. $\mathrm{H}_{2} \mathrm{O}_{2}$ and $\mathrm{OH}$ are chosen as key species here, as the conversion of $\mathrm{H}_{2} \mathrm{O}_{2}$ to $\mathrm{OH}$ is considered as a rate controlling step for the second stage ignition of hydrocarbon fuels [19]. As shown in Fig. 8, CMChs shows very good performance for the key radicals. It is noted that the scatter in the DNS data is more pronounced as compared with heat release rates, suggesting the correlation between sensible enthalpy with radical species is not as strong as other reacting scalars. However, it leads to very good prediction of the mean reaction rates, primarily because the scatter due to the strong non-linearity in the temperature dependence is suppressed. Similarly to heat release rates, CMCht leads to substantial errors. 


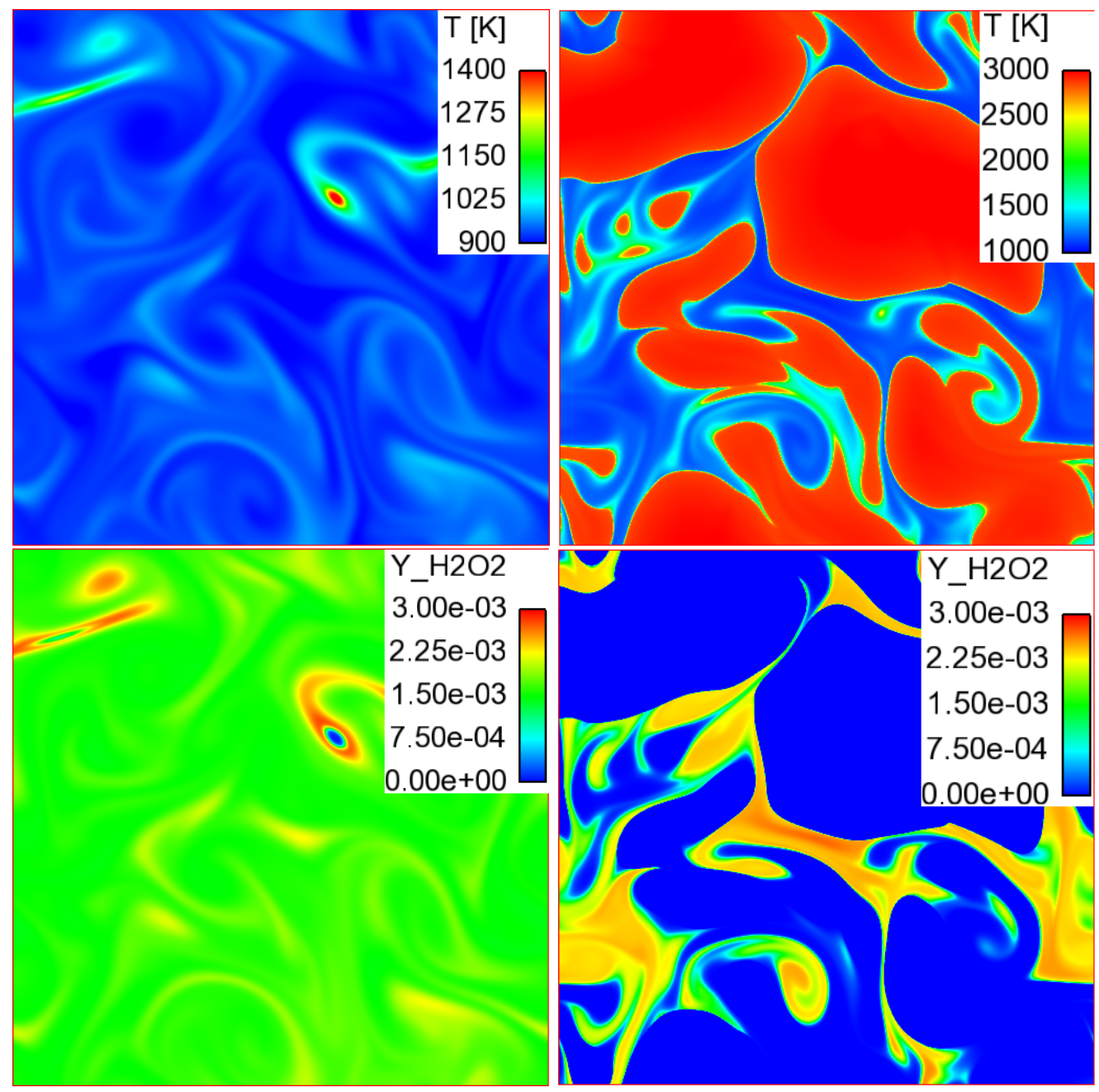

Figure 3: (top) Temperature and (bottom) $\mathrm{H}_{2} \mathrm{O}_{2}$ mass fraction fields in 2-D reduced chemistry DNS of end-gas ignition at two time instances, $2.5 \mathrm{~ms}$ and $2.83 \mathrm{~ms}$ for the Case IV. An ignition spot is seen at the time $2.5 \mathrm{~ms}$ (left). 

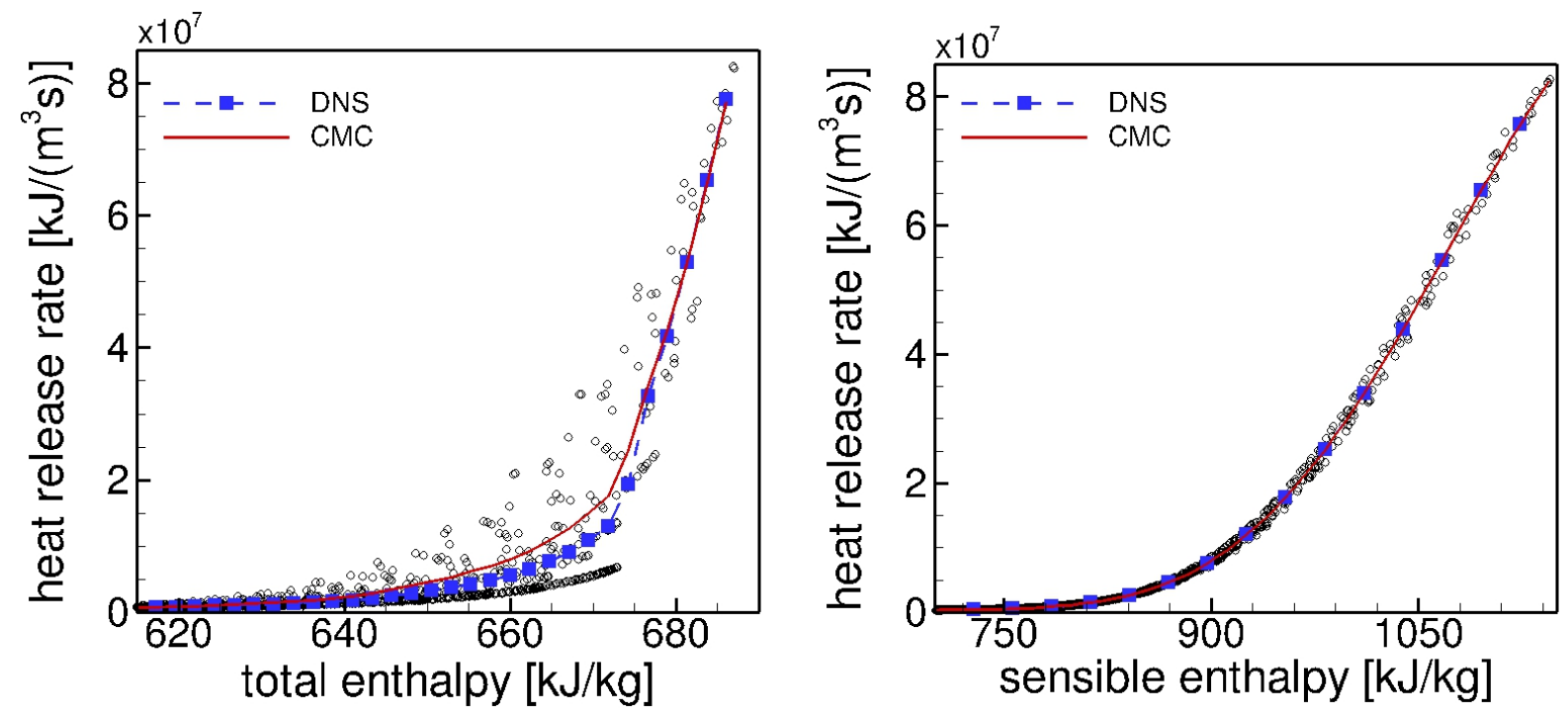

Figure 4: Assessment of CMC in the estimation of heat release rates (Case IV; $t=2.5 \mathrm{~ms}$ ). Heat release rates predicted by (left) CMCht and by (right) CMChs are compared with the DNS data. The blue filled symbols represent the conditional mean heat release rates from the DNS data. The empty symbols denote the heat release rates at DNS grid points.
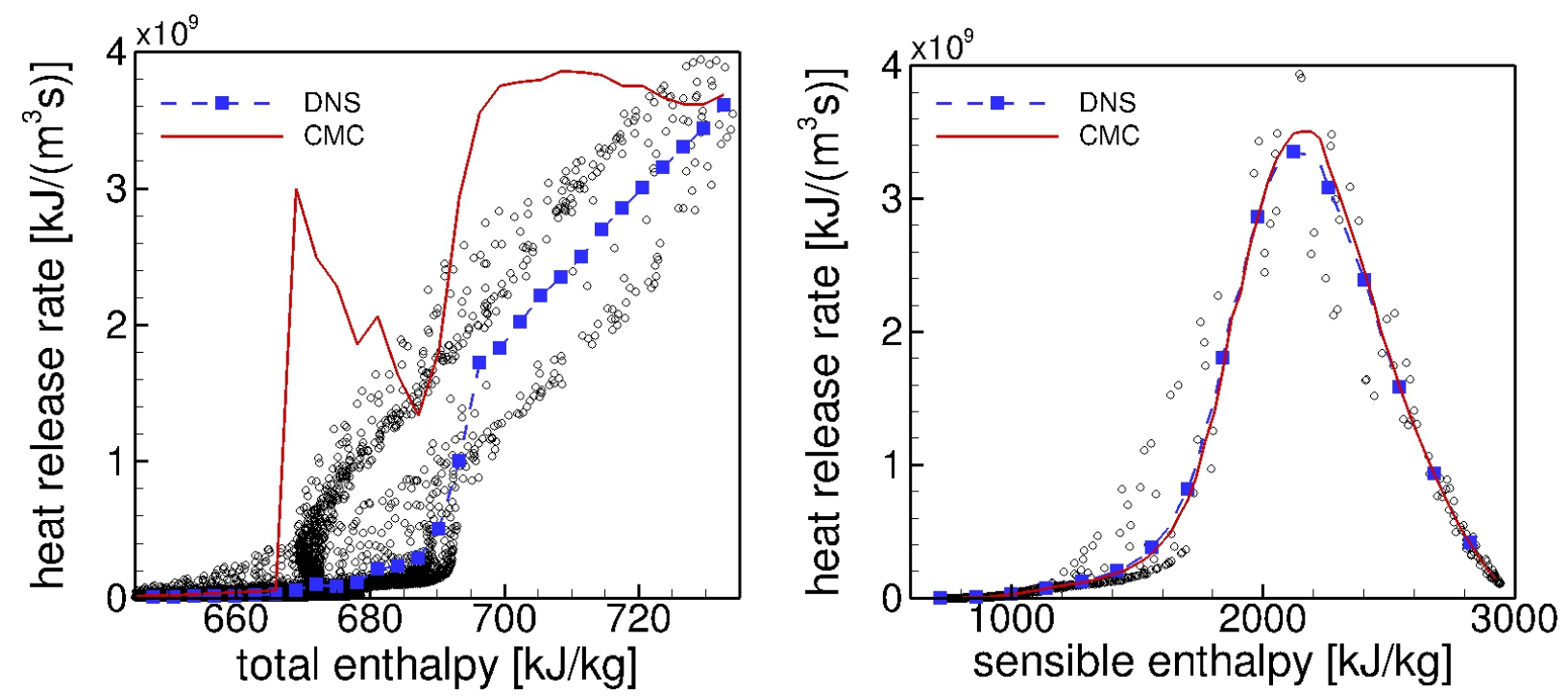

Figure 5: Assessment of CMC in the estimation of heat release rates (Case $1 ; t=2.56 \mathrm{~ms}$ ). Heat release rates predicted by (left) CMCht and by (right) CMChs are compared with the DNS data. The blue filled symbols represent the conditional mean heat release rates from the DNS data. The empty symbols denote the heat release rates at DNS grid points. 

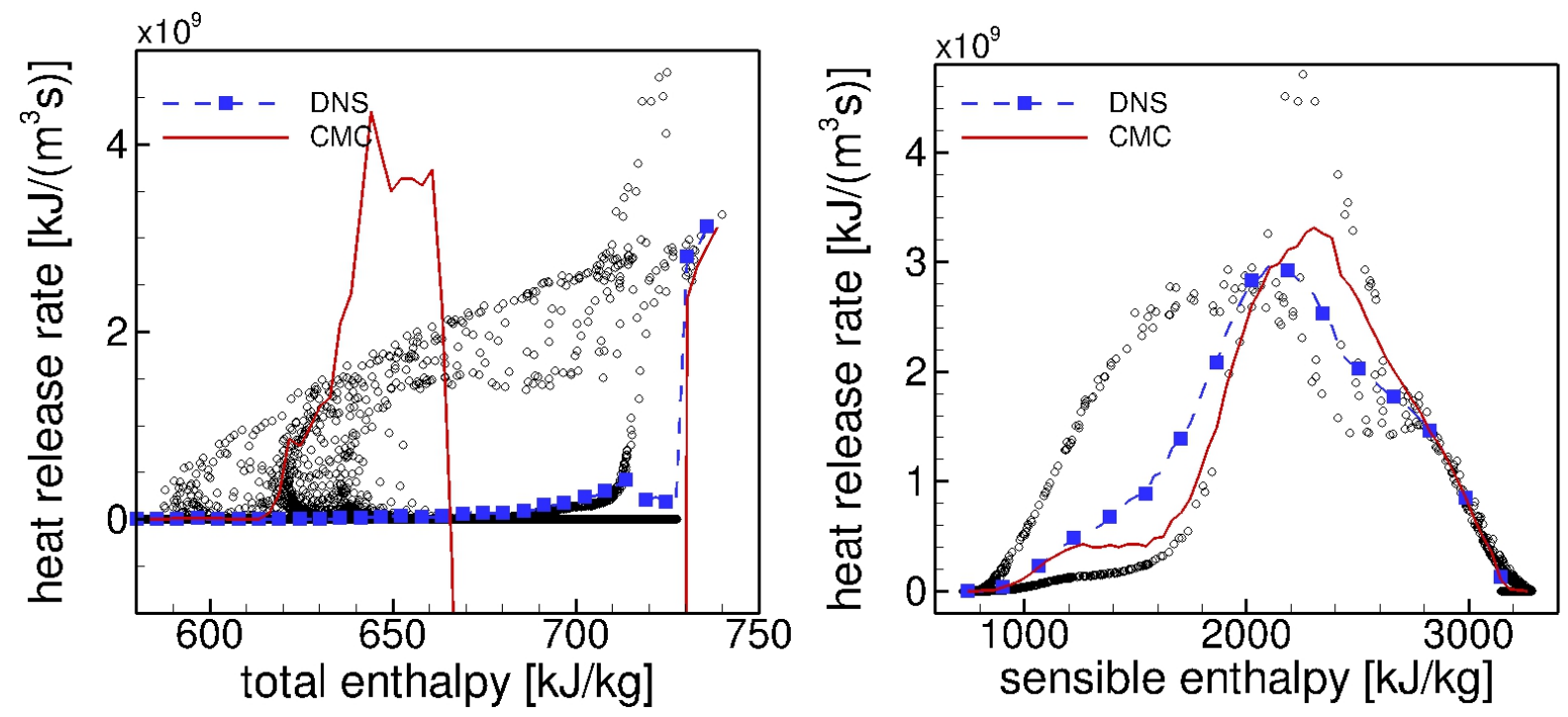

Figure 6: Assessment of CMC in the estimation of heat release rates (Case IV; $t=2.64 \mathrm{~ms}$ ). Heat release rates predicted by (left) CMCht and by (right) CMChs are compared with the DNS data. The blue filled symbols represent the conditional mean heat release rates from the DNS data. The empty symbols denote the heat release rates at DNS grid points.
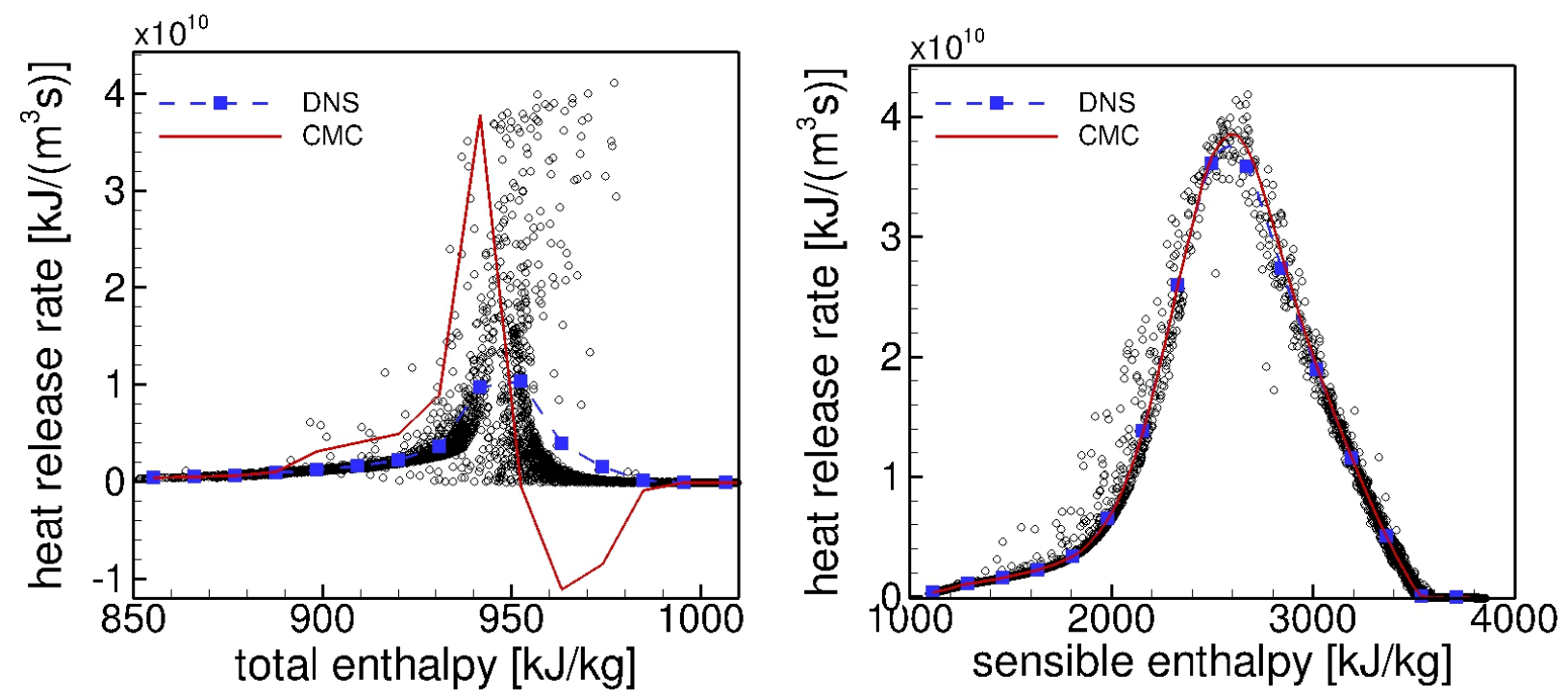

Figure 7: Assessment of CMC in the estimation of heat release rates (Case $1 ; t=2.84 \mathrm{~ms}$ ). Heat release rates predicted by (left) CMCht and by (right) CMChs are compared with the DNS data. The blue filled symbols represent the conditional mean heat release rates from the DNS data. The empty symbols denote the heat release rates at DNS grid points. 


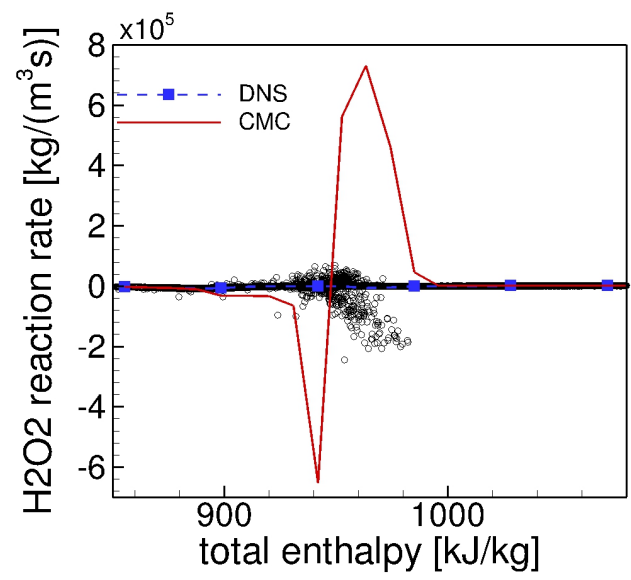

(a)

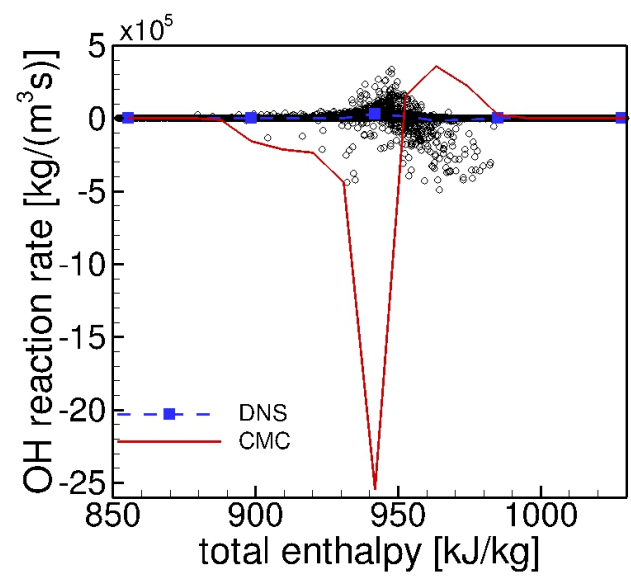

(c)

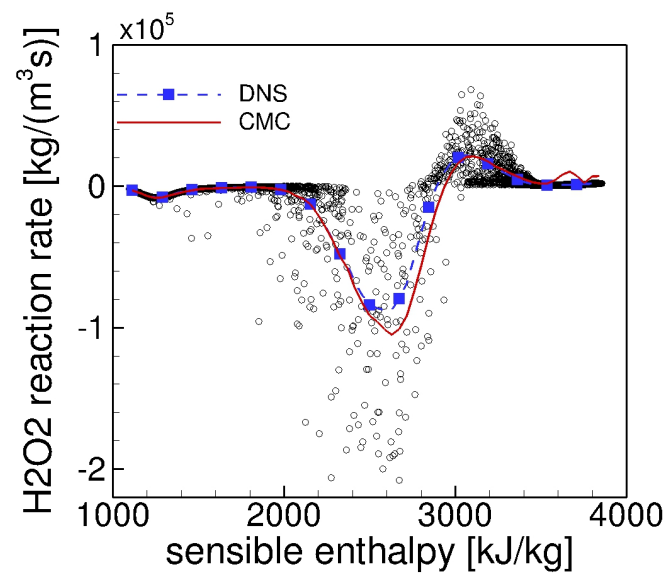

(b)

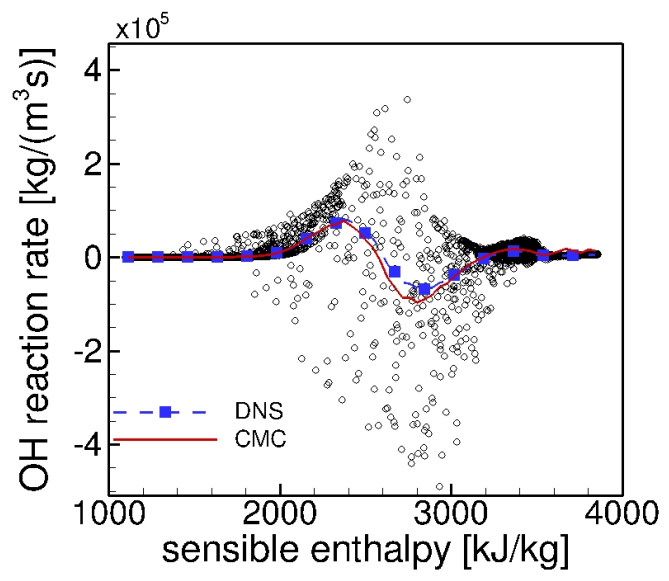

(d)

Figure 8: Assessment of reaction rate estimation for key species. (a) $\mathrm{H}_{2} \mathrm{O}_{2}$ reaction rates predicted by CMCht. (b) $\mathrm{H}_{2} \mathrm{O}_{2}$ reaction rates predicted by CMChs. (c) $\mathrm{OH}$ reaction rates predicted by CMCht. (d) $\mathrm{OH}$ reaction rates predicted by CMChs. The data are from Case 1 when the maximum heat release rates occur, $t=2.84 \mathrm{~ms}$. 


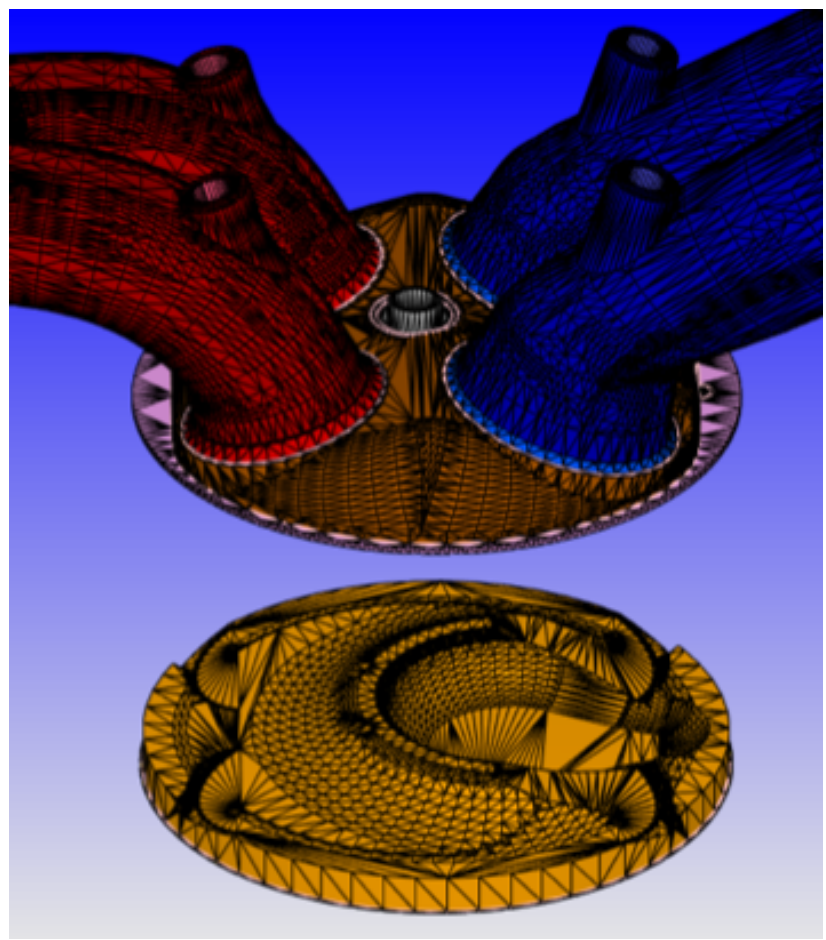

Figure 9: Geometry of the engine used for LES.

In this a priori test, CMChs shows very good performance during the entire duration of ignition processes, while CMCht leads to substantial errors except for very early and late stages of ignition processes. This suggests that the sensible enthalpy is a good conditioning variable in the end-gas ignition problem. The good performance of CMChs in the a priori test comes from the strong correlation of the sensible enthalpy with other reacting scalars, especially temperature.

\subsection{Experimental Campaigns}

This portion of the work occurred at ORNL and utilized a single-cylinder version of the GM LNF 2.0L 4-cylinder design turbocharged gasoline direct injected (GDI) engine from the 2007 Pontiac Solstice. The experimental data for motored, knock, and knock free operation, together with the engine geometry for use in LES (Fig. 9), are provided to the simulation. In what follows, a brief description of the experiments and the representative results is presented.

\section{Operating Conditions and Results}

Several hundred data points were collected with isooctane fueling of SI combustion. Conditions with $0 \%$ and $15 \%$ EGR were collected at several boost and intake temperature conditions. Conditions consisted of $0 \%$ EGR at 1000, 2000, and 3000 rpm engine speeds and 15\% EGR at 1000 and $2000 \mathrm{rpm}$. At each engine speed load was swept at $40^{\circ} \mathrm{C}$ and $90^{\circ} \mathrm{C}$ intake temperatures. Then at 1000 and $2000 \mathrm{rpm}$ at high load ( $20 \mathrm{bar}$ IMEP at $40^{\circ} \mathrm{C}$ ) intake temperature was swept from $40^{\circ} \mathrm{C}$ to $180^{\circ} \mathrm{C}$, or until excessive pre-ignitions occurred. All experiments were with the production engine configuration of 9.2:1 (advertised) compression ratio. The laboratory measurements, though, found the compression ratio of 9.0:1. Isooctane was the fuel used for all conditions. Experiments were designed to explore not only knock limited engine operation across the range of conditions, 


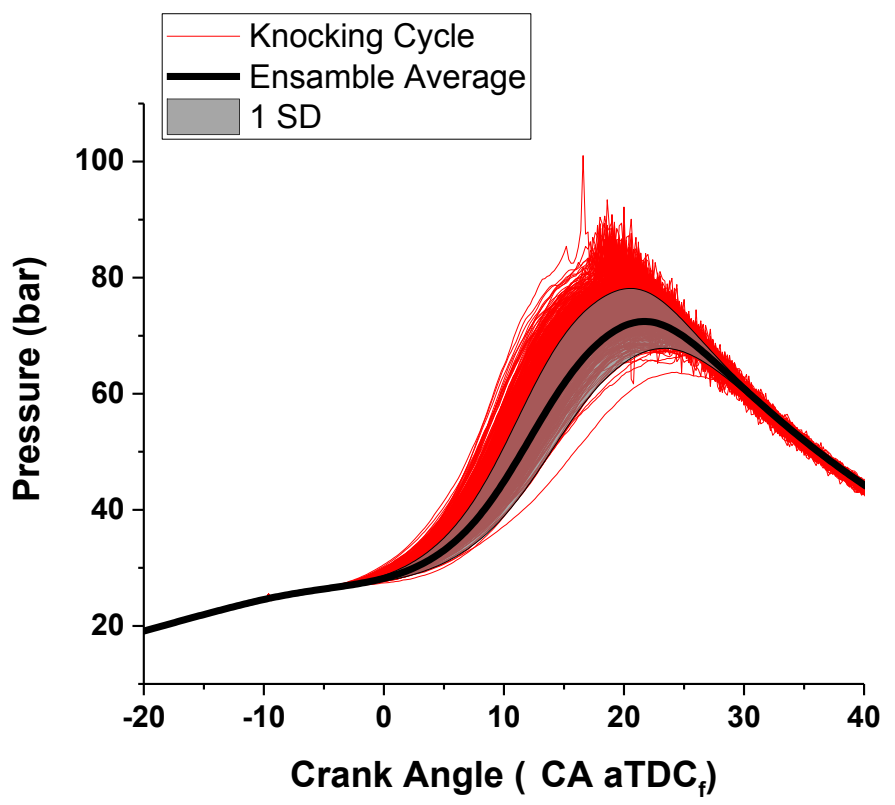

Figure 10: Representative knock limited phasing condition of acquired data from engine experiments .

but also to explore more complex SI combustion effects such as pre-spark heat release (PSHR) and stability limits.

Figure 10 shows the pressure evolution for a representative knocking case (no EGR). Figure 11 highlights the trends in knock limited spark phasing (i.e. CA50), for isooctane at 1000 and $2000 \mathrm{rpm}$ engine speeds, the data shows a piece-wise behavior, where CA50 retard with intake temperature abruptly stops when pre-spark heat release occurs (not illustrated in figure).

Figure 12 shows results of KLSA CA50 phasing at $946 \mathrm{r} / \mathrm{min}$ and $804 \mathrm{~g} / \mathrm{min}$ airflow rates for $0 \%$ and $15 \%$ external cooled EGR. Results show that as load is increased (i.e., increased airflow), EGR has a reduced effectiveness. Specifically, at the $946 \mathrm{r} / \mathrm{min}$ airflow rate, there is no benefit of using $15 \%$ EGR to reduce knock, in fact the KLSA CA50 is actually later with $15 \%$ EGR at this load. However, when the load is reduced EGR shows benefits at reducing knock for intake temperatures below 120C. 


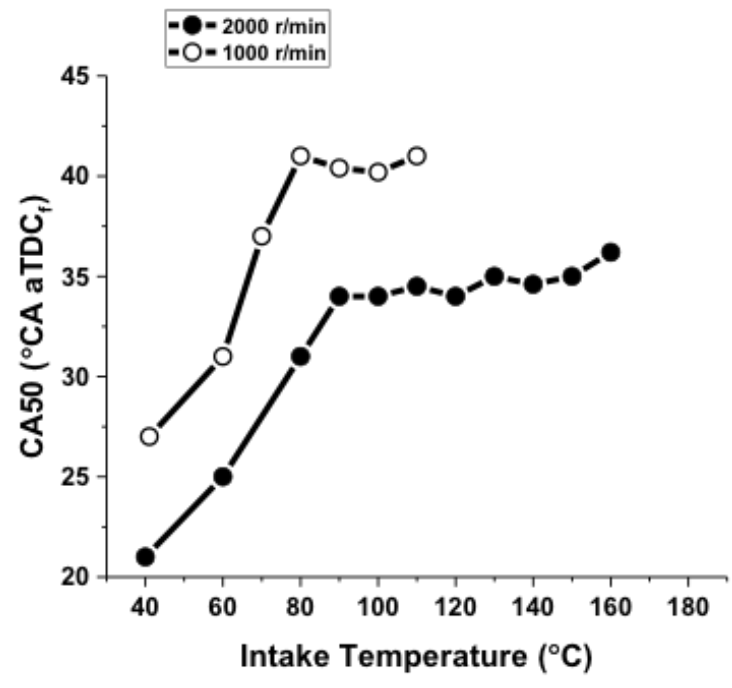

Figure 11: CA50 combustion phasing at the knock limit of isooctane as a function of intake temperature at $2000 \mathrm{rpm}$ and $1000 \mathrm{rpm}$. Operating conditions at each engine speeds were scaled such to provide similar initial conditions, but with increased wall clock time.

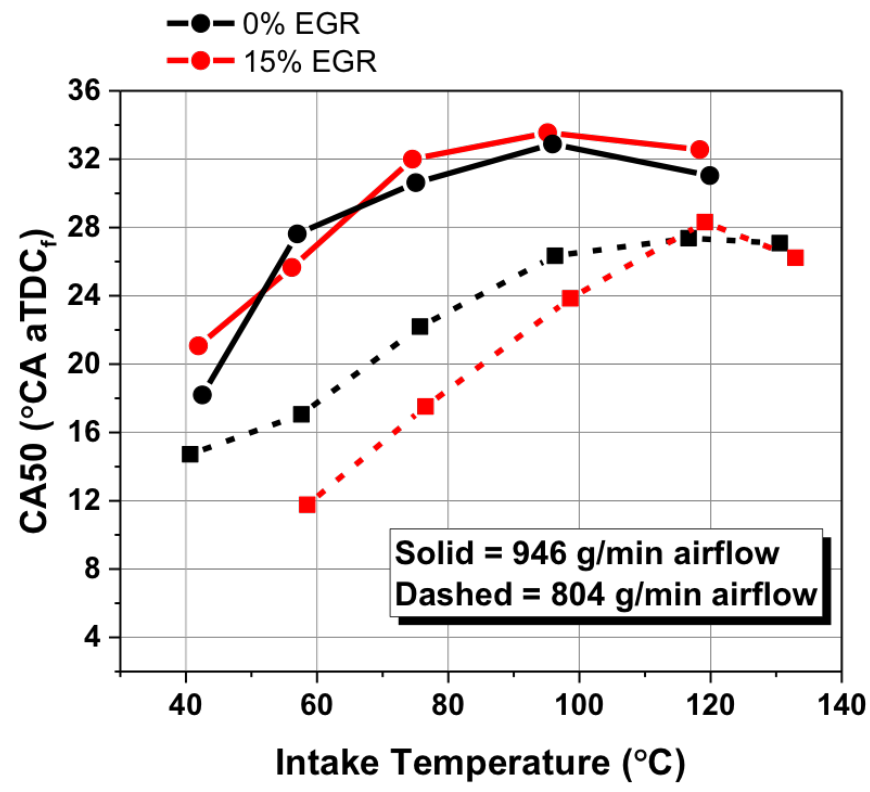

Figure 12: Results of KLSA CA50 phasing at $946 \mathrm{r} / \mathrm{min}$ and $804 \mathrm{~g} / \mathrm{min}$ airflow rates for $0 \%$ and $15 \%$ external cooled EGR. Results show that at elevated loads EGR has no benefit and can make knock worse. 


\subsection{Reproducing Cycle-to-Cycle Variations in the SI Premixed Burning}

Before moving to the validation for knock experiments, the efforts have been made to validate the model and simulation set up for knock-free conditions. The initial efforts lead to, in order to accurately predict the degree of CCVs observed in the experiments, the necessity of developing a laminar-to-turbulent flame transition model that can capture variations of the burning rates during an early stage of spark-ignited flame kernel growth. The modeling framework for sparkignited premixed flame propagation, including FPF, spark ignition, and laminar-to-turbulent flame transition models, is validated for the engine experiments described in the previous section.

\subsubsection{Experiment and Simulation Conditions}

LESs of a non-knocking case of the experiments performed using the GDI engine at Oak Ridge have been performed. The engine specifications and operating conditions are summarized in Table 2 .

The multi-cycle LES was performed using the commercial CFD code CONVERGE v2.3. The FPF model implemented into CONVERGE as UDF is used to describe spark-ignited premixed burning. The laminar-to-turbulent flame transition model described in Sec. 2, as well as other more simplified approaches described below, is used. The one-equation dynamic structure model [20] is used to simulate the turbulent subfilter stresses. The model constants $C_{\epsilon}$ and $C_{k}$ are set to be 0.8 and 0.1 , respectively. The law-of-the wall models are used for wall heat flux and stresses. The Kelvin-Helmholtz (KH) - Rayleigh-Taylor (RT) break-up model [21,22] is used. The collision and coalescence of drops are neglected. A dynamic drag model [23,24] is used. The Frössling model for droplet evaporation [24] is used. The standard values for the spray model parameters are used.

At the computational boundary of the intake manifold, the pressure is set to be the phaseaveraged value from the experiment. The temperature and pressure at the exhaust boundary are also taken from the experiment. The constant wall temperature boundary conditions are used. The wall temperature is set to be $425 \mathrm{~K}, 500 \mathrm{~K}, 450 \mathrm{~K}$, and $600 \mathrm{~K}$ for the intake, the exhaust, the cylinder, and the spark-plug, respectively.

To study the effect of the laminar-to-turbulent flame transition model on CCV prediction, three different simulations named as LTV, LTC, and NLT are preformed. In LTV, the proposed laminarto-turbulent flame transition model is used. In LTC, it is assumed that the transition completes at a fixed size of the kernel. In the formulation, it corresponds to set the transition scale $\eta_{o}$ in Eq, (28) as a constant. The effects of variations in turbulence intensity on the transition are neglected. In NLT, the laminar-to-turbulent flame transition model is not used. The subfilter flame speed model based on local equilibrium is used throughout the kernel growth starting from the spark timing. The Peters model for the subfilter turbulent flame speed, adapted for LES, is used for all the simulations. The parameters associated with the laminar-to-turbulent flame transition model and the subfilter turbulent flame speed model are adjusted to match the simulated in-cylinder pressure with the experimental results. Table 3 shows the parameters used in these three simulations. Multiple cycles are simulated consecutively for LTV. For the simulation of each cycle in LTC and NLT, it is restarted at $-10^{\circ} \mathrm{CAD}$ using the restart files obtained in LTV. As will be discussed in Section 3.3.2, the residual gas effect is very small in the current simulation. It appears that the use of the fields taken from the consecutive cycle LES to test variations in the model does not influence predicted $\mathrm{CCV}$ to a noticeable degree. 


\begin{tabular}{ll}
\hline \hline Bore & $86 \mathrm{~mm}$ \\
Stroke & $86 \mathrm{~mm}$ \\
Compression ratio & 9.0 \\
Fuel & Isooctane \\
Fuel-air equivalence ratio & 1.0 \\
Engine Speed & $2006 \mathrm{RPM}$ \\
Spark timing & $-2.2 \mathrm{CAD}$ \\
Intake Valve Opening (IVO) & $307 \mathrm{CAD}$ \\
Intake Valve Closure (IVC) & $571 \mathrm{CAD}$ \\
Exhaust valve Opening (EVO) & $137 \mathrm{CAD}$ \\
Exhaust Valve Closure (EVC) & $375 \mathrm{CAD}$ \\
Injection start timing & $440.2 \mathrm{CAD}$ \\
Injection end timing & $491.0 \mathrm{CAD}$ \\
Intake pressure & $170 \mathrm{kPa}$ \\
Intake temperature & $312 \mathrm{~K}$ \\
Intake air mass flow rate & $0.986 \mathrm{~g} / \mathrm{cycle}$ \\
\hline \hline
\end{tabular}

Table 2: Engine specifications and operating point. Crank angle degrees (CAD) are relative to combustion phase top dead center (TDC).

\begin{tabular}{lll}
\hline \hline Cases & Transition model & Peters Fame speed model \\
\hline LTV & $k=5, \eta_{c}=1.5 \mathrm{~mm}$ & $b_{1}=9.5, b_{3}=1.3$ \\
LTC & $\eta_{o} \equiv 3.5 \mathrm{~mm}$ & $b_{1}=9.5, b_{3}=1.3$ \\
NLT & None & $b_{1}=9.0, b_{3}=1.0$ \\
\hline \hline
\end{tabular}

Table 3: Parameters for the laminar-to-turbulent flame transition and subfilter flame speed models used in different simulations. 
The cut-cell Cartesian mesh approach is used in CONVERGE to discretize the computational domain. The adaptive mesh refinement (AMR) strategy based on velocity gradient with the refinement criterion of $0.2 \mathrm{~m} / \mathrm{s}$ is employed. The base mesh size used in the simulation is set as $2.4 \mathrm{~mm}$. The mesh size in the cylinder is refined to be $0.6 \mathrm{~mm}$. The AMR mesh size is set to be $0.3 \mathrm{~mm}$. The total number of computational cells during the simulation ranges from $1.8 \mathrm{M}$ to $18 \mathrm{M}$. The simulation was performed on the Owens system in the Ohio Supercomputing Center. 140 cores are used from -130 CAD to 370 CAD while 280 cores are used from 370 CAD to 590 CAD. The computation of a full cycle takes about 64 hours. Twenty cycles have been simulated.

\subsubsection{Results and Discussion}

\section{Flame Characteristics}

Before discussing CCV prediction, the non-dimensional numbers characterizing the burning process are estimated from empirical formulations and LES results. The Karlovitz number $K a$, the Damköhler number $D a$ and the Reynolds number $R e_{t}$ are defined as,

$$
\begin{gathered}
K a=\frac{\tau_{c}}{\tau_{k}}=\frac{l_{F} u_{k}}{l_{k} S_{L}}, \\
D a=\frac{\tau_{t}}{\tau_{c}}=\frac{s_{L} l_{t}}{u^{\prime} l_{F}}, \\
R e_{t}=\frac{u^{\prime} l_{t}}{\nu},
\end{gathered}
$$

where $l_{F}, u_{K}$, and $l_{K}$ are the flame thickness, the Kolmogorov velocity scale, and the Kolmogorov length scale, respectively. $l_{t}, u^{\prime}$ and $\nu$ are the integral length scale, the turbulence intensity, and the kinematic viscosity, respectively. The flame thickness $l_{F}$ is determined from the unburned thermal diffusivity $D_{u}$ and the (unstretched) laminar flame speed $s_{L}$ as $l_{F}=D_{u} / s_{L}$. Following [25], the integral length scale $l_{t}$ is taken as $H / 6$, where $H$ is the clearance height near the cylinder center. $u^{\prime}$, $u_{K}$, and $l_{K}$ are estimated based on the Kolmogorov similarity hypothesis: $u_{K}^{3} / l_{K}=u^{\prime 3} / l_{t}=\epsilon_{\Delta}$ and $u_{K} l_{K}=\nu$. The kinematic viscosity $\nu, s_{L}$, and $\epsilon_{\Delta}$ are taken as the mean value within a sphere with a radius of $3 \mathrm{~mm}$ near the spark plug over 20 LES cycles at the spark timing. Table 4 shows the estimated values of the parameters. The estimated $K a$ suggests that the flame is in the corrugated flamelet regime $(K a<1)$. It is to be noted that when the unburned thermal diffusivity and the laminar flame speed are used to estimate the flame thickness, the resulting value is smaller than the actual thickness. When the flame thickness defined as the ratio of the temperature difference across the flame to the maximum temperature gradient is used, the Karlovitz number for the current case is about 0.5 . When the turbulence intensity is estimated as a half of the mean piston speed, the Karlovitz number becomes 1.33. All these estimations suggest that the flame under the present condition is in or near the corrugated flamelet regime. This indicates that the proposed laminar-to-

turbulent flame transition model, which assumes that the flame is in the corrugated flamelet regime, is a plausible approach for the present case.

\section{$\underline{\text { CCV }}$}

Figure 13 compares the predicted in-cylinder pressure with the experimental measurements. In 


\begin{tabular}{lll}
\hline \hline & Unit & Value \\
\hline$l_{t}$ & {$[\mathrm{~mm}]$} & 2.70 \\
$u^{\prime}$ & {$[\mathrm{m} / \mathrm{s}]$} & 1.49 \\
$S_{L}$ & {$[\mathrm{~m} / \mathrm{s}]$} & 0.76 \\
$l_{F}$ & {$[\mu \mathrm{m}]$} & 3.29 \\
$K a$ & {$[-]$} & 0.11 \\
$D a$ & {$[-]$} & 415 \\
$R e$ & {$[-]$} & 1981 \\
\hline \hline
\end{tabular}

Table 4: Turbulence and flame parameters near the spark plug at the spark timing.

the experiment, the in-cylinder pressure initially decreases after TDC and then rises until reaching the peak value. This behavior is due to the spark timing. In the current case, the spark timing is only $2.2 \mathrm{CAD}$ before TDC. The initial burning rate and the associated pressure rise are not strong enough to counter-balance the effects of volume expansion during the expansion stroke. After the initial phase, the in-cylinder pressure begins to rise as the kernel grows and the burning rates increase. Such characteristics in the initial pressure evolution are well captured by LTV and LTC, both of which consider the laminar-to-turbulent flame transition. The degree of the initial pressure decrease is less in NLT, which neglects the transition. NLT appears to over-predict the burning rate in the early stage. The range of pressure variations predicted by LTV is comparable to that in the experimental measurements. It is much smaller in LTC and NLT. This indicates that CCV can be under-predicted when the variation in the transition process or the transition itself is neglected. It is also noticed that the results of LTV and LTC show a consistently higher peak pressure compared with the experimental observations, similar to the engine LES result with CONVERGE in Zhao et al. [26]. This is probably because the flame-wall interaction is not considered in the present LES.

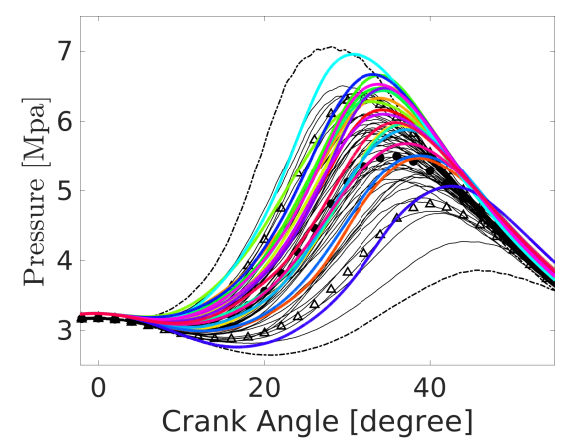

(a) LTV

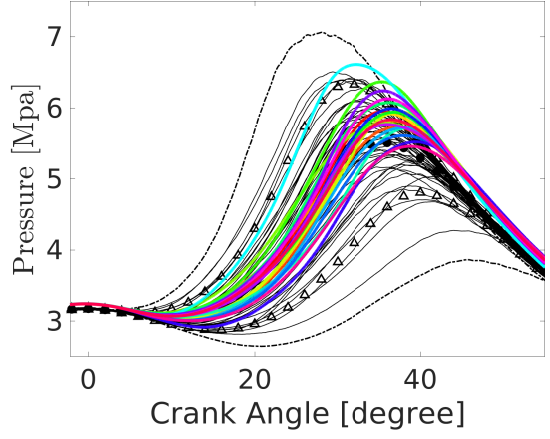

(b) LTC

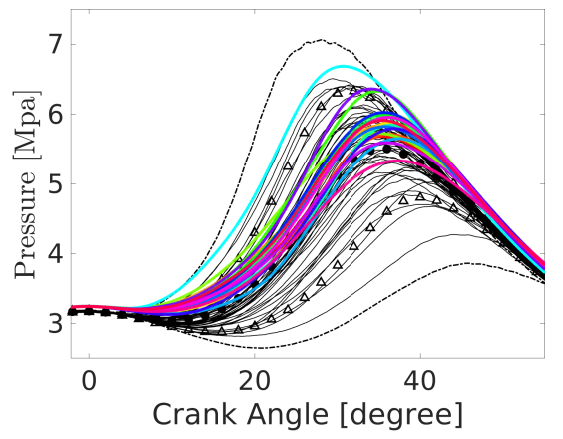

(c) NLT

Figure 13: Predicted in-cylinder pressure in multi-cycle LES (black thin lines: 50 cycles from the experiment, colored thick lines: 20 LES cycles, dashed-dotted lines: fastest and slowest cycles in the experiment, triangles: $5 \%$ and $95 \%$ bounds from 5000 cycles in the experiment, circles: mean pressure in the experiment).

Figure 14 compares the coefficient of variance (COV) of the predicted maximum pressure (Pmax) 
and of the burn-rate-related parameters (CA10, CA50, and CA90) with those from experimental measurements. Here, COVs in the experiment are evaluated for 5000 cycles. 20 cycles are used for the LES results. Overall, the COVs predicted by LTV compare well with the experimental data, with the difference being $-0.61 \%, 0.80 \%,-0.67 \%$, and $-1.20 \%$ for Pmax, CA10, CA50, and CA90, respectively. This result is better than that reported in the previous quarter. For LTC and NLT, COVs for all the quantities considered are under-predicted.

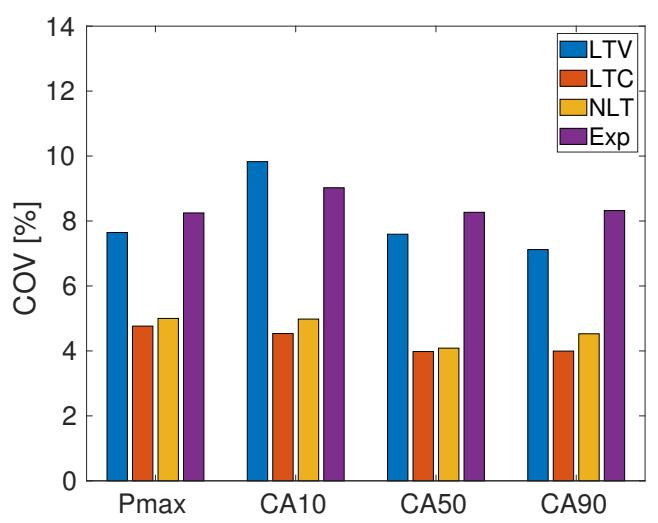

Figure 14: Comparison of the predicted COVs for the maximum pressure, CA10, CA50, and CA90 with the experiment data.

The statistical convergence for COV is checked and the results are shown in Fig. 15. For LTC and NLT, COV of the maximum pressure $P_{\max }$ saturates after about 12 cycles, suggesting that increasing the number of cycles won't improve the predicted CCV by these approaches. The experimental data shows that after about 15 cycles, the COV is converged to a reasonable degree. LTV shows a similar trend to the experiment.

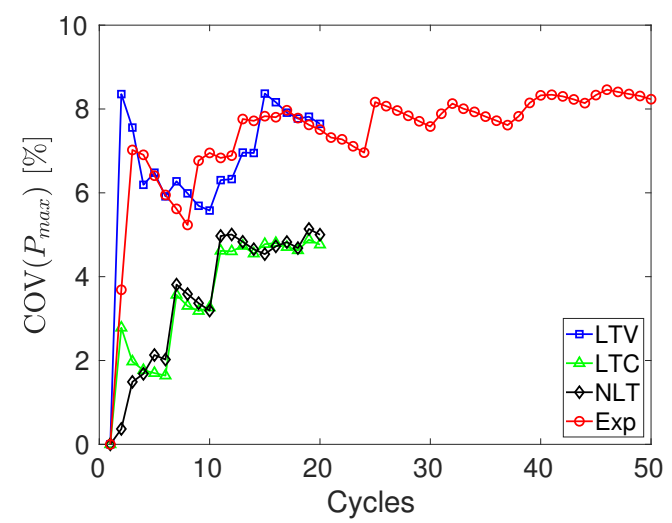

Figure 15: Convergence of COV of the maximum pressure from experiment and from LES.

Figure 16 displays the cycle-by-cycle evolution of CA10, CA50, and CA90 from the experiment and that from LES with LTV. For the experimental data, 50 consecutive cycles are used. In terms of mean values, LTV predicts CA10 and CA50 well but under-predicts CA90. The underprediction is possibly due to neglecting the flame/wall interaction near the cylinder wall in the 
current work, which can reduce the flame speed at the late stage of the flame development. In both the experiment and LTV, the variation of the combustion phasing exhibits a stochastic behavior with respect to the cycle number. No evident correlation between consecutive cycles is noticed. This suggests that using the fields obtained from LTV to restart the simulation of each cycle in LTC and NLT is acceptable.

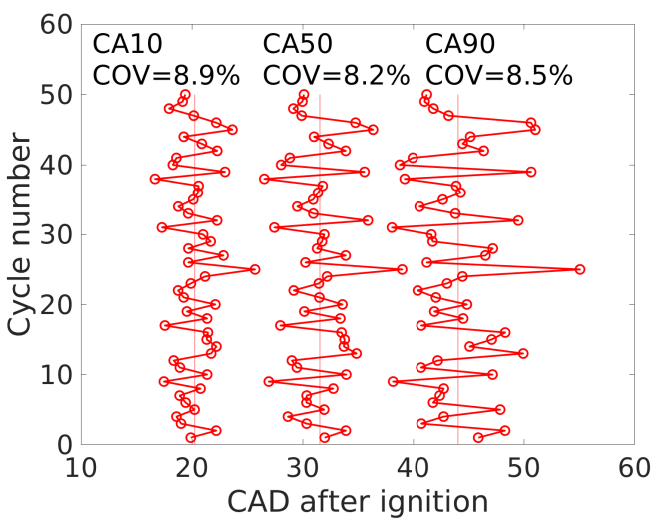

(a)

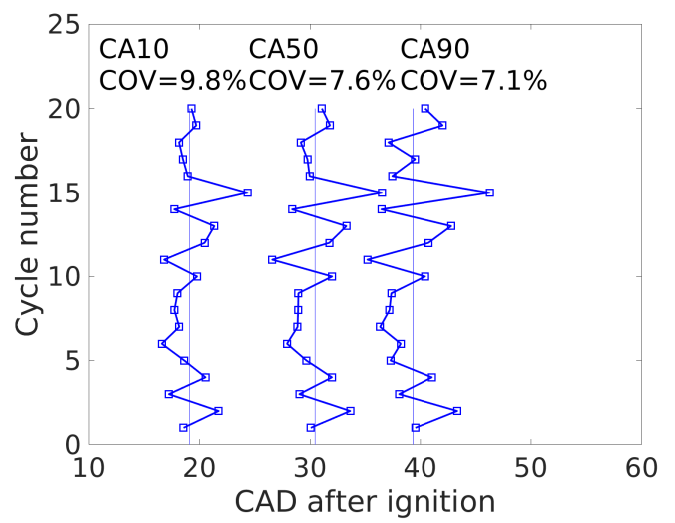

(b)

Figure 16: Cycle-to-cycle variations of CA10, CA50, and CA90 from the experiment(a) and $\operatorname{LTV}(b)$.

\section{Analysis of CCV and Laminar-to-turbulent Flame Transition}

Figures 17, 18, and 19 show the resolved flame surfaces for the four fast and four slow cycles at 10 CAD after TDC for LTV, LTC, and NLT, respectively. The resolved flame surface is represented as the iso-scalar surface with $\tilde{c}=0.5$, where $\tilde{c}$ is the density-weighted filtered progress variable. The fast (slow) cycles denote the cycles that have a high (low) peak pressure. The flame kernels in most cycles show a tendency to move towards the exhaust side of the cylinder due to the tumbling flow motions $[27,28]$. The fast and slow cycles in the simulation do not show a clear difference in terms of the flame shape. This is in contrast to the observations by Zhao et al. [26], where the flame shape was found to be related to large scale flow structures. For LTV, there are substantial variations in the flame kernel sizes for the fast and slow cycles. In contrast, the difference in the kernel size is much smaller in LTC and NLT, which is consistent with the small COVs predicted by those two approaches. It is also noticed that the slow cycles in NLT generally have larger kernels than those in LTV and NLT at 10 CAD. This is because the neglect of the laminar-to-turbulent flame transition in NLT leads to the over-predicted sub-filter flame speed $s_{c, t}$ in the early stage of the kernel growth.

Figure 20 shows the temporal evolution of the resolved flame surface area $A_{\text {res }}$ and the surface averaged sub-filter flame speed $\left\langle s_{c, t}\right\rangle$ from LTV, LTC, and NLT. $A_{r e s}$ and $\left\langle s_{c, t}\right\rangle$ are estimated as,

$$
\begin{gathered}
A_{\text {res }}=\int_{V}|\nabla \tilde{c}| d v, \\
\left\langle s_{c, t}\right\rangle=\frac{\int_{V} s_{c, t}|\nabla \tilde{c}| d v}{\int_{V}|\nabla \tilde{c}| d v} .
\end{gathered}
$$




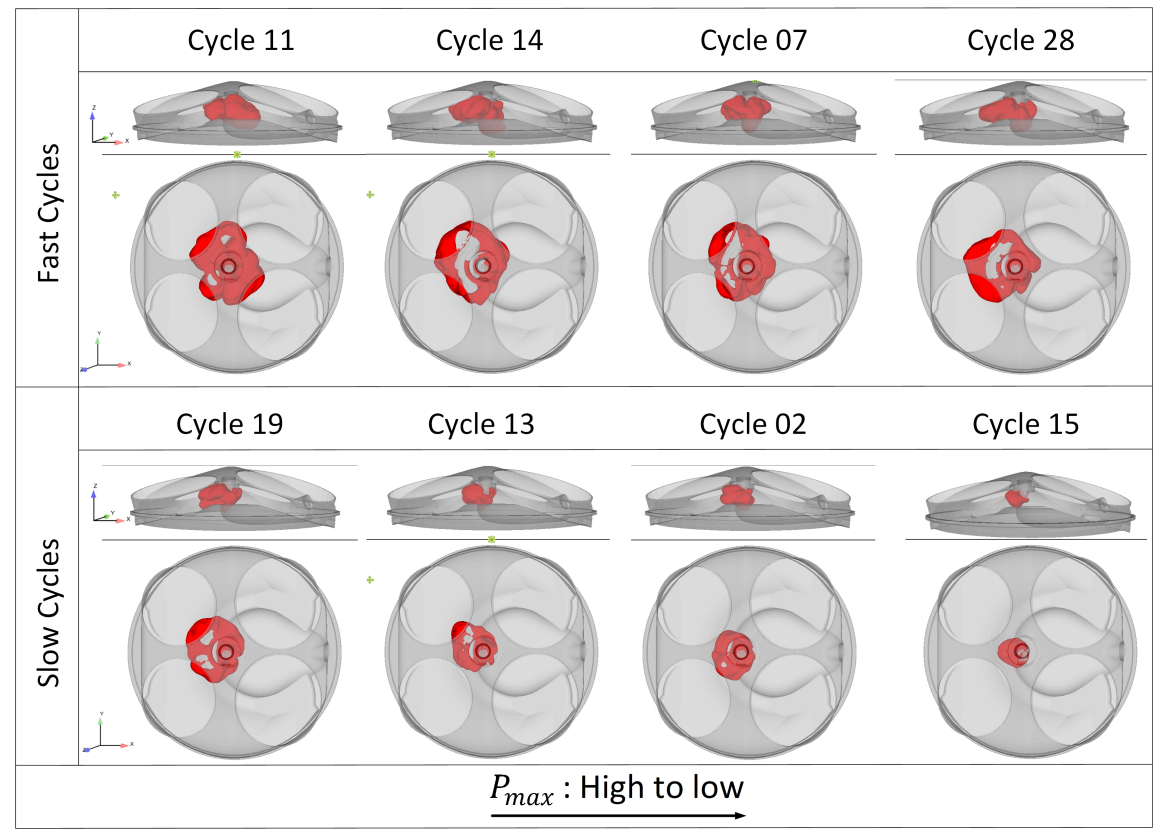

Figure 17: Flame kernels in the LTV simulations. The resolved flame surfaces represented by the iso-scalar surfaces with $\tilde{c}=0.5$ at $10 \mathrm{CAD}$ after TDC for the four fast cycles and the four slow cycles are shown. The intake ports are on the right and the exhaust ports are on the left.

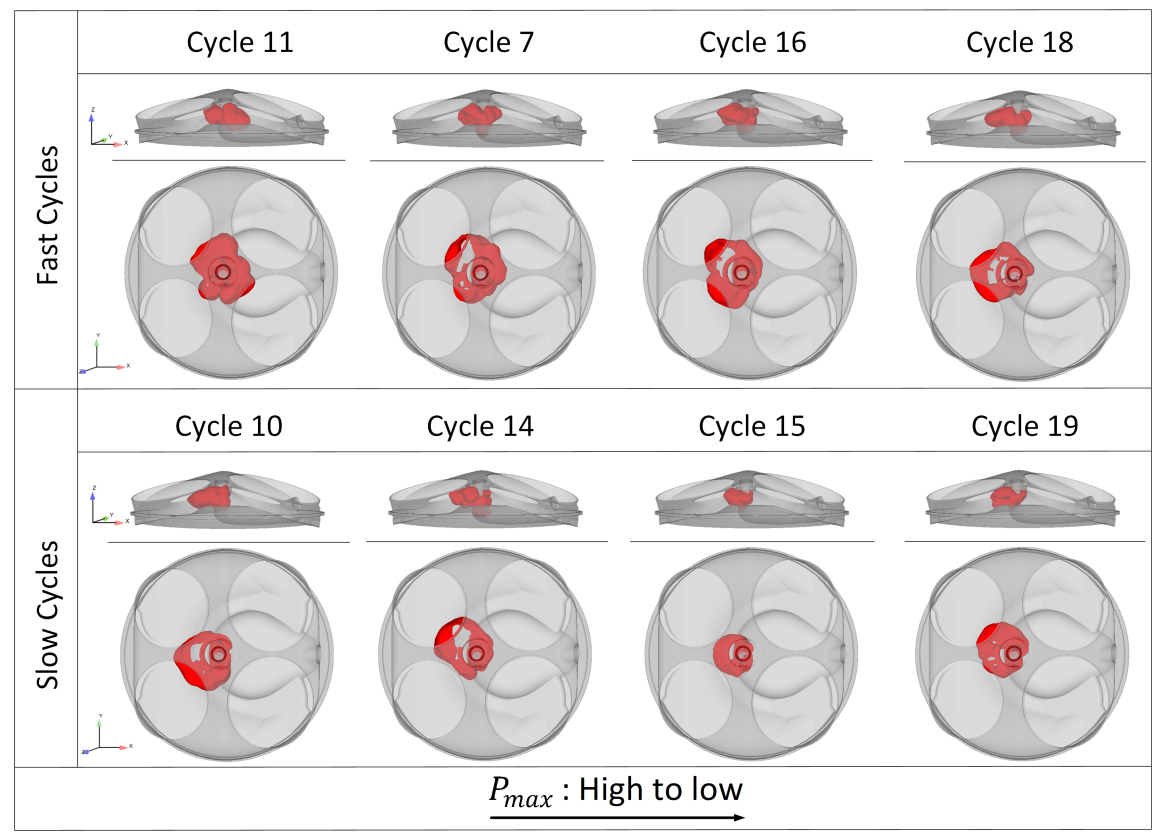

Figure 18: Flame kernels in the LTC simulations. The resolved flame surfaces represented by the iso-scalar surfaces with $\tilde{c}=0.5$ at $10 \mathrm{CAD}$ after TDC for the four fast cycles and the four slow cycles are shown. The intake ports are on the right and the exhaust ports are on the left. 


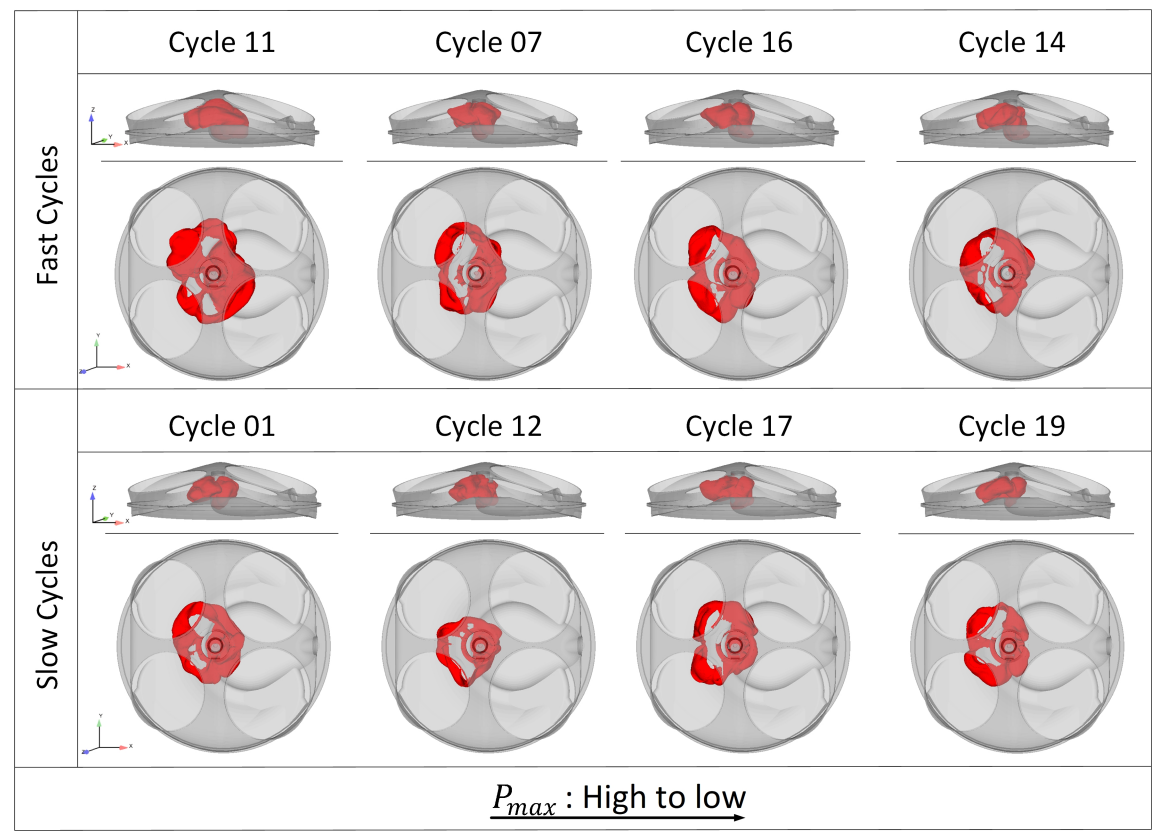

Figure 19: Flame kernels in the NLT simulations. The resolved flame surfaces represented by the iso-scalar surfaces with $\tilde{c}=0.5$ at $10 \mathrm{CAD}$ after TDC for the four fast cycles and the four slow cycles are shown. The intake ports are on the right and the exhaust ports are on the left.

The overall shape of the time evolution of the resolved flame surface area is similar to that of the flame surface area reported in previous experimental works $[12,13]$. It is clearly seen that the degree of variations in the resolved flame surface area in LTV is larger than those in LTC and NLT. It is also noticed that the resolved flame surface area grows more rapidly initially (from the spark timing to 5 CAD after TDC) in NLT than in LTV and LTC. The results are consistent with those in Figs. 17-19 for the visualized flame surface at 10 CAD. For each cycle before spark discharge, the flow and scalar fields are identical for all three simulations, since the restart file taken from the LTV simulation at that time is used for the LTC and NLT simulations. Therefore, the difference in the variations in the flame development comes only from the laminar-to-turbulent flame transition model. As can be observed in Figs. 20(d)-(e), the sub-filter turbulent flame speed, $\left\langle s_{c, t}\right\rangle$, in both LTV and LTC increases from the laminar flame speed to the equilibrium turbulent sub-filter flame speed as the flame kernel develops. The flame transition process in LTV is smooth. The transitional process is not captured in NLT, where the equilibrium sub-filter flame speed is applied from the spark timing. The over-predicted sub-filter flame speed in NLT results in the more rapid kernel growth and the less obvious initial drop of the in-cylinder pressure as shown in Fig. 13(c). With the effects of turbulence on the flame transition being captured, LTV predicts larger variations of $\left\langle s_{c, t}\right\rangle$ than LTC, where the transition occurs at a fixed kernel size, and NLT, where the transition is not considered. The variations in the sub-filter flame speed $\left\langle s_{c, t}\right\rangle$ lead to those in the kernel growth rate. As shown in Figs. 20(d)-(f), the predicted resolved flame surface area in LTC and NLT show smaller variations than in LTV, consistent with CCV of in-cylinder pressure.

Figure 21 shows the correlation between CA10 and CA02 and between CA02 and the transition duration time $\tau_{\text {lam-turb }}$, respectively. Here, the transition time $\tau_{l a m-t u r b}$ is defined as the temporal 

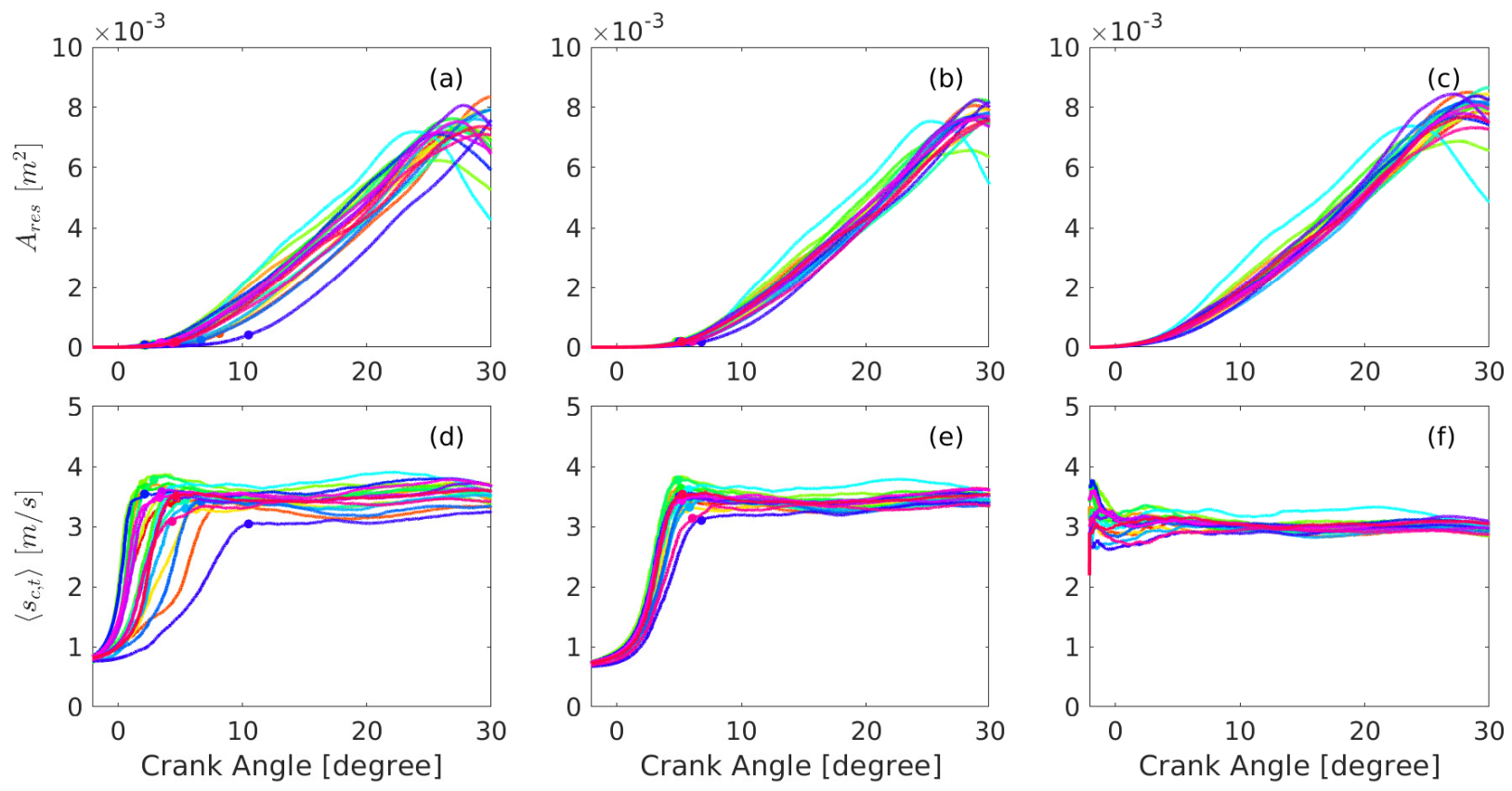

Figure 20: Temporal evolution of the sub-filter flame speed $\left\langle s_{c, t}\right\rangle$ and the resolved flame surface area $A_{r e s}$ for LTV, LTC, and NLT $\left(\left\langle s_{c, t}\right\rangle\right.$ : (a)-(c); $A_{r e s}$ : (d)-(f); LTV: (a) and (d); LTC: (b) and (e); NLT: (c) and (f); the circular markers represent the points when the laminar-to-turbulent flame transition ends).

duration in CAD between the spark timing and the time when the laminar-to-turbulent flame transition ends. Both the experiment and LES show that the initial flame propagation (CA02) strongly affects the overall burning rate, which is consistent with previous findings [12, 28, 29]. Moreover, the strong correlation between CA02 and $\tau_{\text {lam-turb }}$ shown in Fig. 21(b) suggests that the modeling of the laminar-to-turbulent flame transition plays an important role in determining the predicted $\mathrm{CCV}$ in the present case. In LTC, the variation in $\tau_{\text {lam-turb }}$ is much smaller than that in LTV and thus causes the significantly smaller CCV.

\section{Variations in Laminar-to-turbulent Flame transition}

Considering the importance of capturing the variation of the laminar-to-turbulent flame transition in engine LES, it is of interest to study the source for this variation in the proposed model, LTV. There are two possible sources for the variation in the flame transition: the sub-filter flame speed $s_{c, t}$ and the development of the resolved flame kernel. The sub-filter flame speed represents the effects of small-scale turbulence, as well as the laminar flame speed, on the burning rate, while the resolved flame surface area represents the effects of the large-scale flow field. In LTV, the sub-filter flame speed $s_{c, t}$ depends on the transition scale $\eta_{o}$, the laminar flame speed $S_{L}$, and the equilibrium sub-filter flame speed $s_{c, t}^{e q}$. As discussed in Sec. ??, the transition scale $\eta_{o}$ is affected by the laminar flame speed $S_{L}$ and the sub-filter dissipation rate $\epsilon_{\Delta}$. The equilibrium sub-filter flame speed $s_{c, t}^{e q}$ depends on both $S_{L}$ and the small scale turbulence fields. The wrinkling of the resolved flame surfaces is influenced by the large scale flow structures, which can be represented by the 


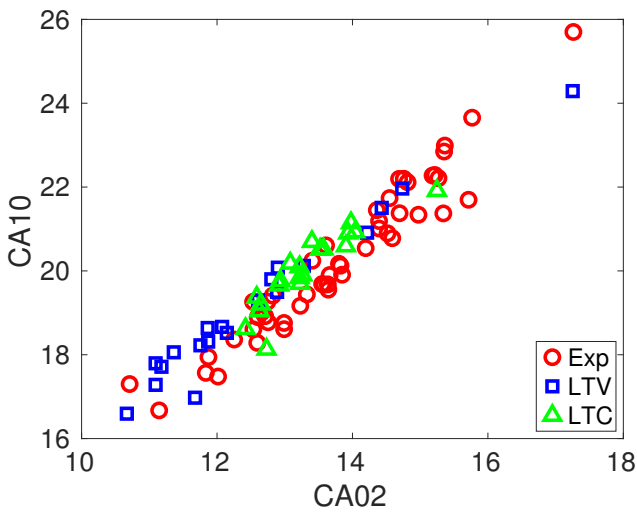

(a)

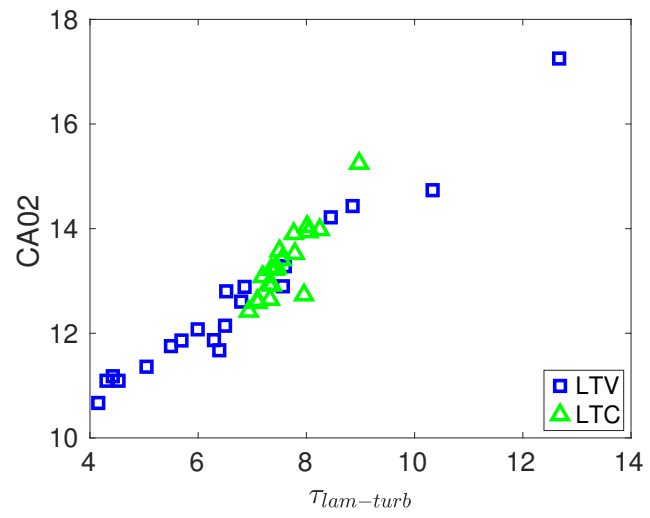

(b)

Figure 21: Correlation between CA10 and CA02(a) and between CA02 and the laminar-toturbulent flame transition time $\tau_{\text {lam-turb }}(\mathrm{b})$.

resolved velocity components, $\tilde{u}, \tilde{v}$, and $\tilde{w}$. Based on the above, the key parameters chosen to analyze the sources for the variations in the early kernel development are $\tilde{u}, \tilde{v}, \tilde{w}, S_{L}$, and $\epsilon_{\Delta}$. The relative importance of these parameters to the transition process is analyzed through a multi-variant regression analysis. The five variables are volume-averaged within a sphere near the spark-plug at the spark-timing. The radius of the sphere chosen for volume averaging is $3 \mathrm{~mm}$. This size is close to the average flame kernel size at which the laminar-to-turbulent flame transition ends. When the size of volume used for averaging is too small, the evaluated parameters may not be representative. The radius of $5 \mathrm{~mm}$ has also been tested, and the results are found to be similar to those using the $3 \mathrm{~mm}$ sphere.

The present multi-variant regression analysis takes the following steps. Firstly, the single regression analysis between the transition time $\tau_{\text {lam-turb }}$ and each parameter is performed, from which the parameters that are weakly correlated with $\tau_{l a m-t u r b}$ are identified and eliminated from the multi-variant regression. The criterion for weak correlation is chosen as $|R|<0.1$, where $R$ is the correlation coefficient. Then, a multi-variant regression analysis [28] is performed. In the multi-variant analysis, the transition time $\tau_{\text {lam-turb }}$ is assumed as a linear function of multiple parameters: $\tau_{\text {lam-turb }}(n)=\sum_{i=1}^{p} \beta_{i} X_{i}(n)+\epsilon(n)$, where $n, X_{i}(n), \beta_{i}$, and $\epsilon$ represent the cycle number, each parameter of interest at the cycle number, the coefficient, and the residual, respectively. $\beta_{i}$ and $\epsilon$ are determined using the least squares fitting. The degree of correlation between the parameter $X_{i}$ and the transition time $\tau_{\text {lam-turb }}$ is examined by calculating the correlation coefficient between $X_{i}$ and $\tau_{\text {lam-turb }}-\sum_{k \neq i}^{p} \beta_{k} X_{k}$, discarding the contribution of the other parameters. The degree of correlation between the actual transition time $\tau_{\text {lam-turb }}$ and its linear regression, $\sum_{i=1}^{p} \beta_{i} X_{i}(n)+\epsilon(n)$, is also examined.

Figure 22 shows the results from the single regression analysis. The resolved large-scale velocity components $\tilde{u}$ and $\tilde{w}$ are found to be weakly correlated with the transition time $\tau_{\text {lam-turb }}$. The small scale turbulence, which is represented by $\epsilon_{\Delta}$, is found to be the most important source for the variation in the transition process, as expected. The resolved velocity component $\tilde{v}$ and the laminar flame speed $S_{L}$ also play a role in causing variations in the flame transition in the present case. The velocity component $\tilde{v}$ contributes to move the flame kernel in the direction away from 
the solid walls associated with the spark plug.
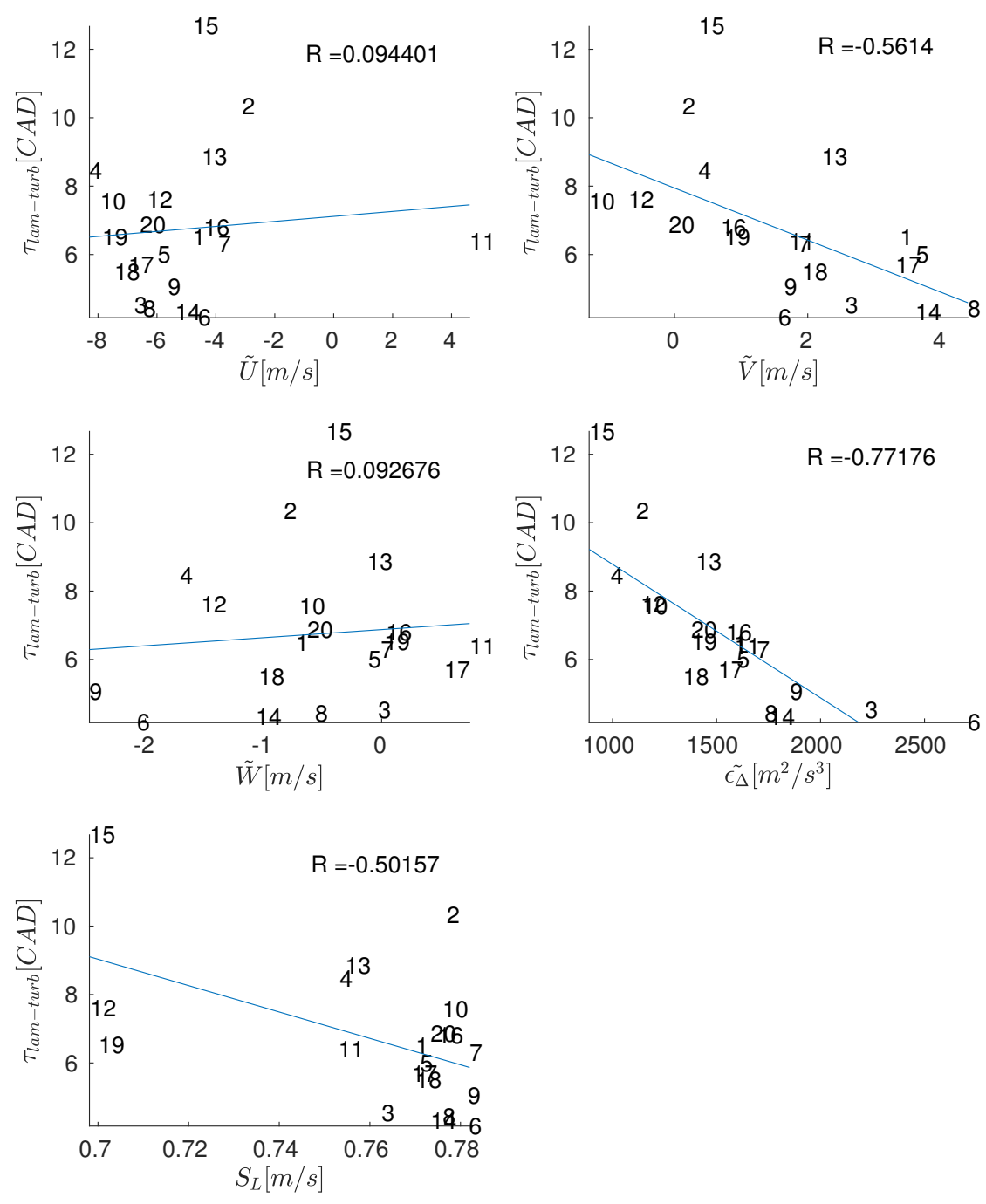

Figure 22: Results from the single-variant regression analysis. $\mathrm{R}$ denotes the correlation coefficient. The numbers inside the plot windows represent the cycle number and their locations indicate the values of the transition time and a parameter for the given cycle.

Figure 23 shows the correlation coefficients obtained from the multi-variant regression analysis. The correlation coefficient between $\tau_{\text {lam-turb }}$ and its linear regression is 0.81 , which seems to indicate that the derived multi-linear regression is a sufficiently good representation. The correlation between $\tau_{\text {lam-turb }}$ and the parameters confirms the findings in the single regression analysis. The level of correlation for the small scale turbulence is higher than for the large scale velocity effect and the laminar flame speed. The negative correlation between $\tau_{l a m-t u r b}$ and $\tilde{v}$ may be because the convective motions with large values of $\tilde{v}$ tend to move the flame kernel away from the 
spark-plug walls and thus promote the growth of the flame kernel. The effects of the convective motion can be seen in Fig. 24, which shows the locations of the flame kernels for two selected cycles when their size reaches $3 \mathrm{~mm}$. The flame kernel in the fast cycle (Cycle 14) is in less contact with the walls associated with the spark plug than in the slow cycle (Cycle 10).

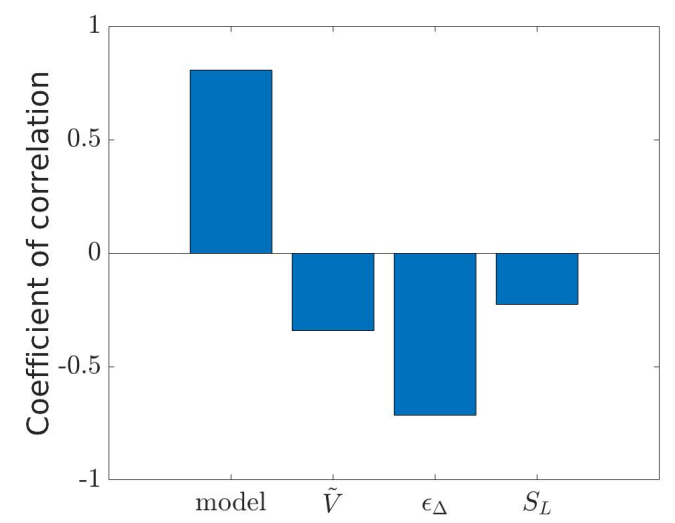

Figure 23: Correlation coefficients obtained from multi-variant regression. The term "model" denotes the derived multi-variant regression approximation.

The factors causing the variation of the laminar flame speed are also investigated. Figure 25 shows the variation of the laminar flame speed $S_{L}$ as a function of the unburned temperature $T_{u}$, the dilution species mass fraction $Y_{d i l}$, and the equivalence ratio $\phi$. The stratification in the mixture composition or the spatial inhomogeneity of $\phi$ is related to the injection and evaporation of the fuel and its mixing with air. The thermal stratification in the unburned mixture or the spatial inhomogeneity of $T_{u}$ results from fuel evaporation and mixing, wall heat transfer, and the residual gas effect. The fraction of the residual gas is represented by $Y_{d i l}$ in the present stoichiometric operation with no exhaust gas recirculation (EGR). In Fig. 25, the variation in the laminar flame speed is caused mainly by the stratification of the equivalence ratio and the thermal stratification in the unburned mixture. For the present case, the mass fractions of the residual gas components are small $\left(\sim O\left(10^{-2}\right)\right)$. As a result, the effects on the laminar flame speed are minor, as can be seen for the laminar flame speed obtained by the empirical formula in Fig. 25 (center). 


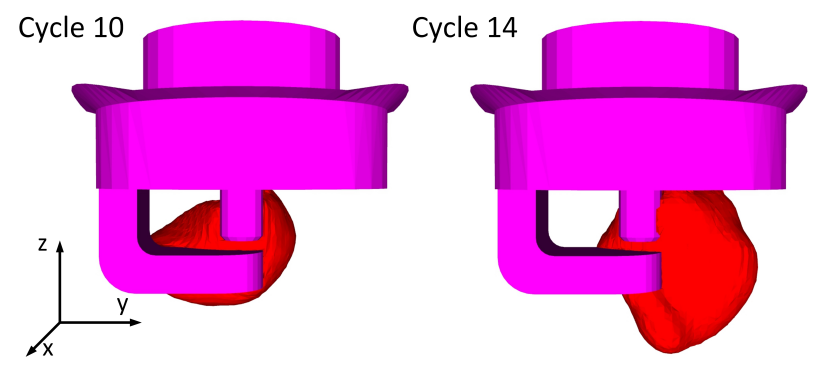

Figure 24: Spark plug and the location of a flame kernel when its size reaches $3 \mathrm{~mm}$. The results for a slow cycle (Cycle 10) and a fast cycle (Cycle 14) are shown (purple: spark plug; red: iso-scalar surface with $\tilde{c}=0.5$ ).
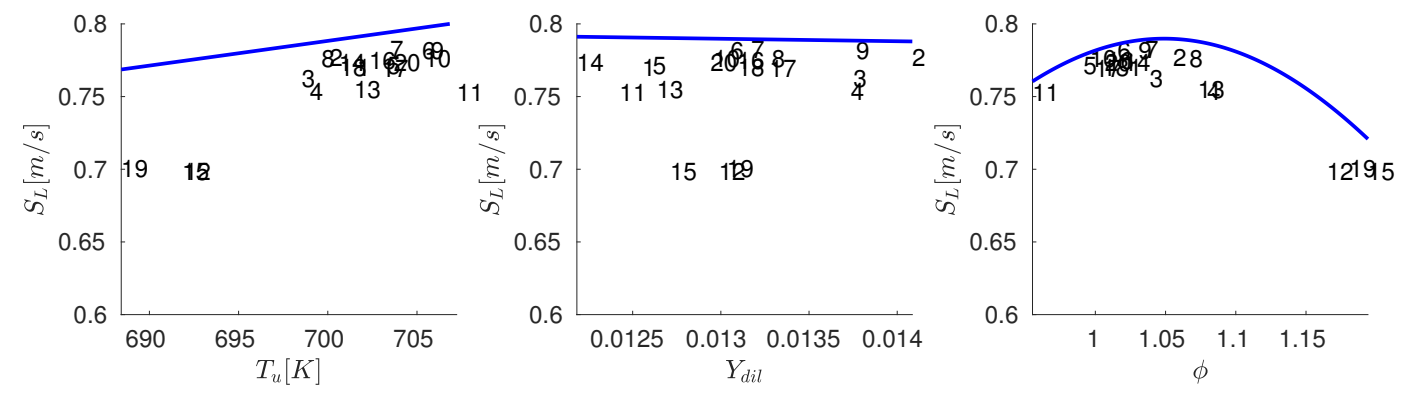

Figure 25: Dependence of the laminar flame speed on the unburned temperature $T_{u}$ (left), the dilution species mass fraction $Y_{\text {dil }}$ (center), and the equivalence ratio $\phi$ (right). The numbers inside the plot windows represent the cycle number and their locations indicate the values of the laminar flame speed and a parameter for the given cycle. The blue line denotes the laminar flame speed estimated using Gülder's laminar flame speed formula [30], in which the average values are used for other parameters than a parameter investigated in a plot. 


\subsection{Knock Model Validation with Experimental Data}

Multicycle LES of a knocking case have been conducted and the results are compared with the experimental data to validate the knock model.

\subsubsection{Stoichiometric Operation}

The engine specification and the conditions for validation cases from the Oak Ridge experiments under the stoichiometric operation are summarized in Tables 5 and 6, respectively. The case R018 is the reference case. For the cases R019 and R020, the spark timing is retarded, while keeping all other conditions the same. For the cases in Table 6, there is no EGR.

\begin{tabular}{ll}
\hline \hline Compression ratio & 9.0 \\
Engine Speed (RPM) & 1000 \\
Fuel & Iso-octane \\
Equivalence ratio & 1.0 \\
Spray injection timing $(\mathrm{CAD})$ & 440, duration 35 \\
Spray temperature $\left({ }^{\circ} \mathrm{C}\right)$ & 53.3 \\
IVO, IVC $(\mathrm{CAD})$ & 308,600 \\
EVO, EVC $(\mathrm{CAD})$ & 130,408 \\
Intake air pressure $($ bar $)$ & 1.41 \\
Intake air temperature $\left({ }^{\circ} \mathrm{C}\right)$ & 39 \\
\hline \hline
\end{tabular}

Table 5: Engine specifications for the ORNL experiments under the stoichiometric operation.

\begin{tabular}{ll}
\hline Case & ST (CAD) \\
\hline R018 & -2.99 \\
R019 & -1.59 \\
R020 & -0.19 \\
\hline
\end{tabular}

Table 6: Spark timing for the ORNL validation cases under the stoichiometric operation.

\section{Computational Models and Simulation Details}

To describe end-gas ignition, the spatially-integrated total-enthalpy-based CMC model is used. The reduced chemical mechanism for PRF [10,11], which consists of 121 species and 538 reactions, is used. The total-enthalpy-based CMC knock model is activated at $30 \mathrm{CAD}$ bTDC, when the mean temperature in the end-gas region reaches around $600 \mathrm{~K}$. It is assumed that the reaction activity below this temperature has negligible influence on the knock. The transport equation model [31] for the subfilter variance and the associated scalar dissipation rate modeling based on the time scale ratio between velocity and scalar are used to obtain the filtered scalar dissipation rate $\widetilde{N}$. The conditional scalar dissipation rate $\langle N \mid \eta\rangle^{e}$ in Eq. (8) is evaluated by averaging the filtered scalar dissipation rate with the condition that the normalized enthalpy has a particular value, $\eta$. The averaging is taken over the whole end-gas region. The FDF of the normalized enthalpy is 


\begin{tabular}{ll}
\hline \hline Subgrid-scale stresses & One-equation dynamic structure model \\
Injection & Blob injection \\
Break-up & KH (Kelvin-Helmholtz)-RT (Rayleigh-Taylor) model \\
Vaporization & Frössling model \\
Drop drag & Dynamic drop drag model \\
Drop-wall interaction & Wall-film model \\
Premixed flame propagation & FPF model \\
End-gas auto-ignition & Spatially-integrated CMCht model \\
\hline \hline
\end{tabular}

Table 7: Physical models used in LES.

assumed to have the beta distribution parametrized by the filtered mean and subfilter variance of the enthalpy, which are solved in CONVERGE. 7 major species are solved for in CONVERGE. The hybrid coupling method in Eq. (13) is used for the coupling between the CONVERGE flow solver and the CMC solver. The threshold of the maximum conditional heat release rate for the transition of the coupling method (from Eq. (15) to Eq. (14)) and the conditioning variable (from total enthalpy to the pseudo-total-enthalpy) is taken as $10 \mathrm{~kJ} /\left(\mathrm{m}^{3} \mathrm{~s}\right)$. The bounds of the conditioning variable are clipped based on its probability to reduce the influence of the region with the very low probability to those with higher probability. The values outside the probability range from $5 \times 10^{-4}$ to $1-5 \times 10^{-4}(0.05 \%$ at each end $)$ are excluded.

The FPF method is used to described spark-ignited premixed flame propagation. The laminarto-turbulent flame transition model used for the present cases is not the final version described in the previous subsection [32], since it was not available at the time of performing the simulations. The difference is the use of the volume-averaged Gibson scale, instead of the surface-averaged one, when evaluating the effects of turbulence on the transition process. The model constants used in the sub-filter flame speed model of Peters are $b_{1}=10.0$ and $b_{3}=1.6$. The model constants in the laminar-to-turbulent flame transition model are $\eta_{0}=0 \mathrm{~mm}$ and $k=2.15$. This difference is not expected to affect knock model validation as long as CCVs in the pressure are reasonably well captured.

The physical models used for subfilter turbulence and sprays are summarized in Table 7 . The dynamic structure model is used for the subgrid-scale turbulent stresses. The model parameters in the one-equation model are $C_{k}=1.0$ and $C_{\epsilon}=0.8$. The fuel injection is modeled with the "Blob" injection method [33], and the initial sizes of the droplets are determined based on the nozzle diameter. The Kelvin-Helmholtz (KH) and Rayleigh-Taylor (RT) models [21,22] are used to simulate spray break-up. A dynamic drag model [23,24] is used for the drop drag calculation. The evaporation of the sprays is modeled using the Frössling model [24]. A wall-film model [34] is used for the spray-wall interaction. The discharge coefficient for liquid injection is determined to match the injection pressure, 100 bar. The other model parameters are unchanged from the standard values.

Constant temperature boundary conditions are used for the walls. The wall temperatures for different surfaces are summarized in Table 8 . The wall temperature conditions used are determined based on the previous works on the knock simulation $[10,35,36]$. For the temperature for the exhaust valves, which affect knock behaviors more, the value used in other LES studies ranges 


\begin{tabular}{ll}
\hline \hline Cylinder head & $450 \mathrm{~K}$ \\
Cylinder liner & $450 \mathrm{~K}$ \\
Piston crown & $450 \mathrm{~K}$ \\
Spark plug & $600 \mathrm{~K}$ \\
Intake runner & $350 \mathrm{~K}$ \\
Intake valve stem & $450 \mathrm{~K}$ \\
Intake valve face & $550 \mathrm{~K}$ \\
Exhaust runner & $725 \mathrm{~K}$ \\
Exhaust valve & $725 \mathrm{~K}$ \\
\hline \hline
\end{tabular}

Table 8: Wall temperature boundary conditions.
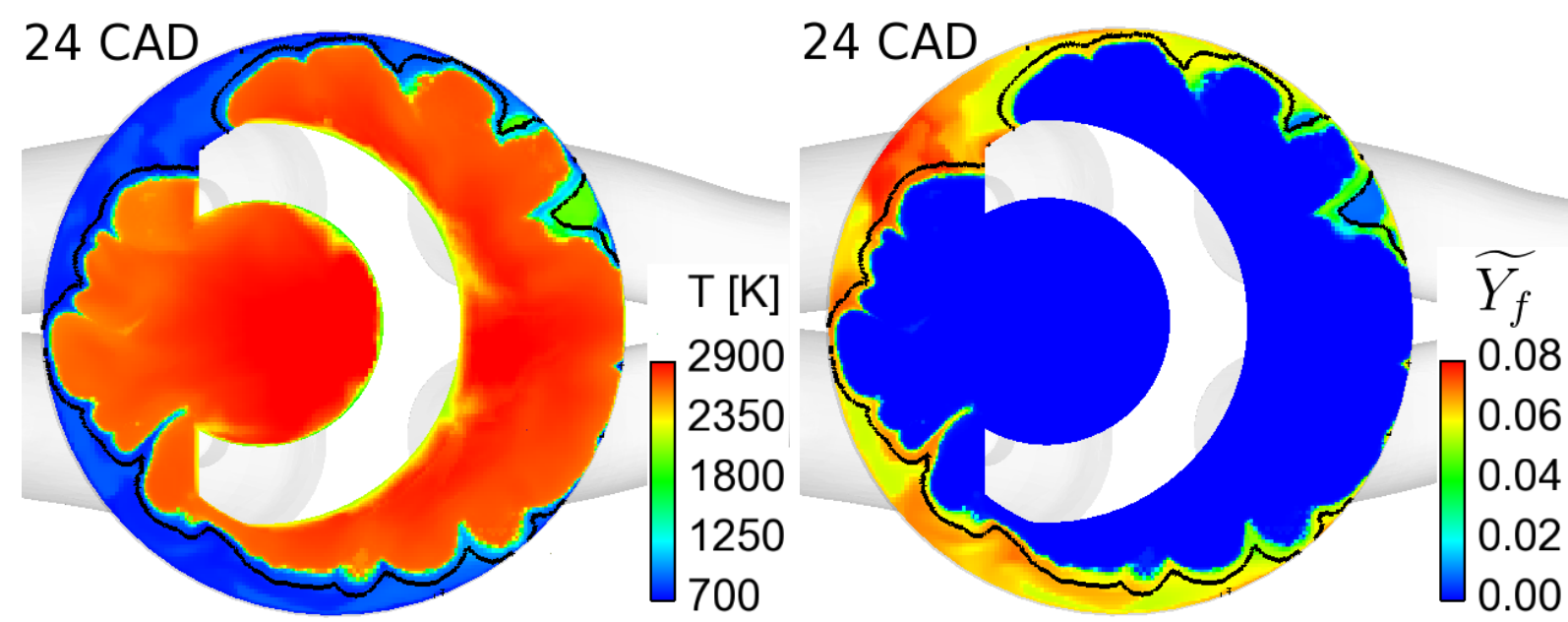

Figure 26: Temperature (left) and fuel mass fraction (right) fields at 24 CAD aTDC for a knocking cycle (Cyc8) for the case R018. The black lines indicate the premixed flame fronts.

from around $690 \mathrm{~K}$ to about $785 \mathrm{~K}[35,36]$. In the present study, the temperature on the exhaust valve wall is set to be $725 \mathrm{~K}$ and the sensitivity study on the exhaust valve temperature is performed.

The base mesh size is $2.4 \mathrm{~mm}$, the AMR mesh size is $0.3 \mathrm{~mm}$, and the total number of computational cells ranges from $2.5 \mathrm{M}$ to $8.5 \mathrm{M}$. The dynamic structure model is used for the subfilter turbulence. Fifteen cycles are simulated. The pressure is probed at four different locations in the cylinder to detect knock.

\section{$\underline{\text { Results }}$}

Figure 26 shows the temperature and fuel mass fraction fields at 24 CAD aTDC for a knocking cycle. The ignition spots in the end-gas region, where temperature is higher and fuel mass fraction is lower than in other end gas regions, are seen. End-gas ignition starts occurring at $21 \mathrm{CAD}$ aTDC for this knocking cycle. The pressure fields before and after the end-gas ignition (Fig. 27) show that the stronger pressure waves are generated by the rapid heat release due to the end-gas ignition.

Figure 28 shows the in-cylinder pressure at a probed location for the fifteen cycles. The CCVs are well reproduced using the present models and associated model parameters. Pressure oscilla- 

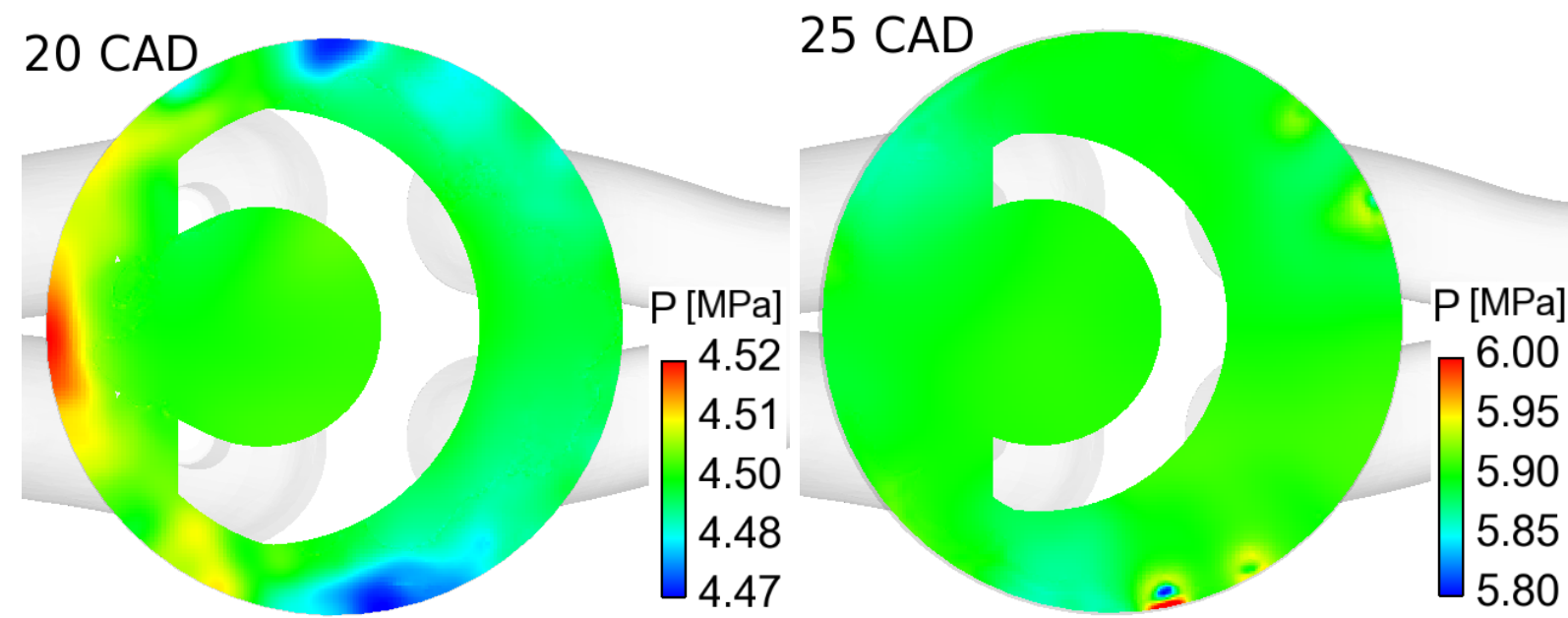

Figure 27: Pressure fields at 20 CAD aTDC and at 25 CAD aTDC for a knocking cycle (Cyc8) for the case R018. End-gas auto-ignition starts occurring at $21 \mathrm{CAD}$ aTDC.

tions are observed in all the cycles. The pressure oscillations in the simulation start at around a time when the pressure peaks, which matches well with those from the experiments. The amplitudes of pressure oscillations are comparable to those in the experimental data. The knock occurrence in the case R018 is captured by the total-enthalpy-based CMC model.
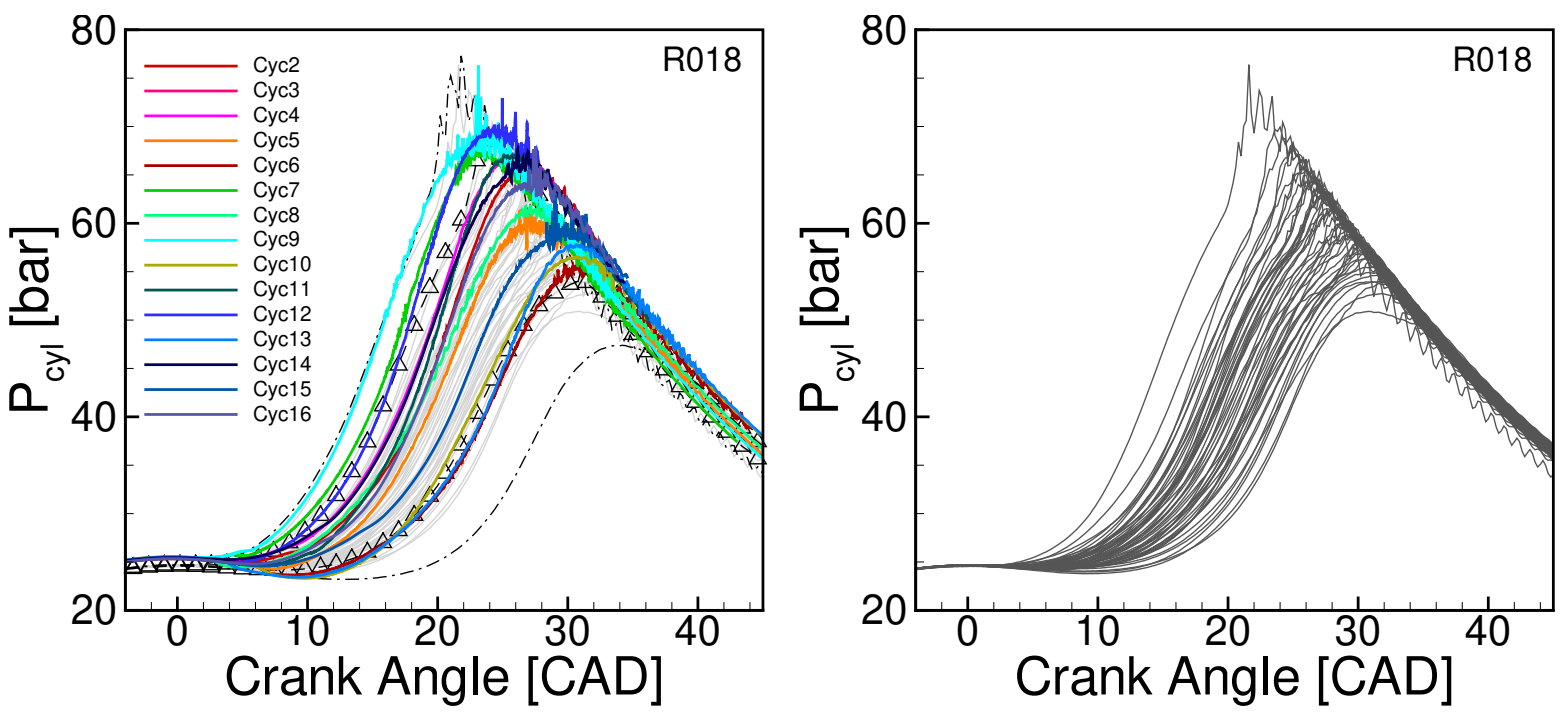

Figure 28: In-cylinder pressure evolution in consecutive multi-cycle LES with total-enthalpy-based CMC for R018 (thin gray lines: measured 50 cycles cylinder pressure randomly selected from 5000 cycles in the ORNL experiment R018, black dashed-dotted lines: fastest and slowest cycles in the experiment, black lines with triangles: $5 \%$ and $95 \%$ bounds from 5000 cycles in the experiment, thick colored lines: pressure from fifteen consecutive LES cycles). In the figures on the right, only measured pressure curves are shown to better present the amplitude of the pressure oscillations in the experiment.

To further validate total-enthalpy-based CMC, the spark timing (ST) is retarded and the results are compared with the corresponding cases R019 and R020. Figure 29 shows the in-cylinder pressure for the selected LES cycles and the corresponding experimental data for R019 and R020. 
Results for R018 are also shown to better present the trend for ST retarding. Three cycles from R018 are selected, one for a fast cycle (Cyc12), one for a slow cycle (Cyc6), and one for in between (Cyc5). The model employed for premixed flame propagation well captures the combustion phasing and pressure evolution when the ST is retarded. The pressure oscillations start around a time when the pressure peaks for the selected cycles in the cases R018-R020. The amplitude of pressure oscillations in the case R020 is apparently smaller than those in R018 and R019 for the selected cycles. However, in the cases R019 and R020, the amplitude of pressure oscillations in LES seems to be larger than those in the experiments. It is to be noted that filtering is applied to the experimental data for pressure, which removes small magnitude high-frequency components.

Figure 30 the effects of the exhaust valve temperature on the knock prediction for the case R020 where the spark timing is the most retarded among the three cases, R018-R020. The temperatures of $610 \mathrm{~K}$ and $690 \mathrm{~K}$ are used in addition to the reference value of $725 \mathrm{~K}$. The sensitivity study is performed for the temperature of the exhaust valve, as it is expected to affect the end-gas ignition more that that of other parts does. For this sensitivity study, for a given selected cycle (Cyc6 or Cyc8), the simulation was resumed at an early intake stroke, after changing the temperature on the exhaust valve. This allows for a sufficient time for the thermal field to adapt to the new boundary condition, while reducing the computational cost by performing LES for a part of a cycle. Since the temperature field developed in the new simulation after changing the boundary condition affects the velocity and turbulence fields, the premixed burning rates and the pressure evolution are dependent on the temperature used for the exhaust valve in a somewhat random manner. On the other hand, it can be seen that the knocking tendency is reduced when the temperature on the exhaust valve is reduced.

\subsubsection{Dilute Operation: Exhaust Gas Recirculation}

\begin{tabular}{ll}
\hline \hline Compression ratio & 9.0 \\
Engine Speed (RPM) & 2000 \\
Fuel & Iso-octane \\
Equivalence ratio & 1.0 \\
Spray injection timing (CAD) & 440 , duration 50 \\
Spray temperature $\left({ }^{\circ} \mathrm{C}\right)$ & 44.8 \\
IVO, IVC $(\mathrm{CAD})$ & 332,624 \\
EVO, EVC $(\mathrm{CAD})$ & 118,396 \\
Spark timing $(\mathrm{CAD})$ & -7.55 relative to combustion TDC \\
Intake air temperature $\left({ }^{\circ} \mathrm{C}\right)$ & 57 \\
Intake air pressure $($ bar $)$ & 1.98 \\
EGR $(\%)$ & 15 \\
\hline \hline
\end{tabular}

Table 9: Engine specifications and operating conditions for the ORNL engine experiment with EGR (Case R023).

Multicycle LES of a knocking case with EGR is performed to further validate the knock model. The engine operating conditions are summarized in Tables 9 and the wall temperature boundary 

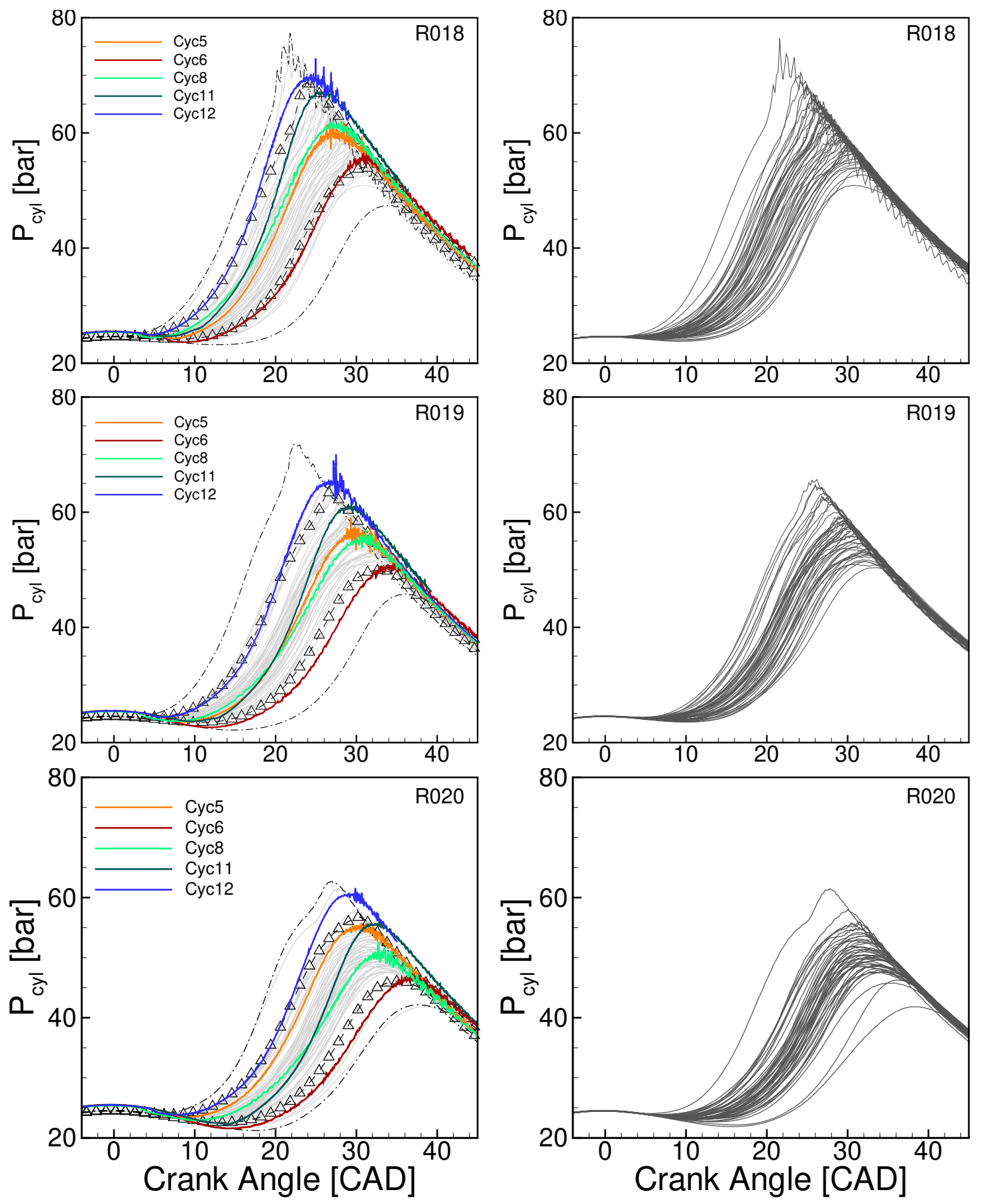

Figure 29: In-cylinder pressure evolution for three selected cycles for the cases R018-R020. The prediction is from consecutive multi-cycle LES with total-enthalpy-based CMC (thin gray lines: measured 50 cycles cylinder pressure randomly selected from 5000 cycles in the ORNL experiments, black dashed-dotted line: fastest and slowest cycles in the experiment, black lines with triangles: 5\% and 95\% bounds from 5000 cycles in the experiment, thick colored lines: pressure from selected consecutive LES cycles). In the figures on the right side, only measured pressure curves are shown to better present the amplitude of the pressure oscillations in the experiment. 

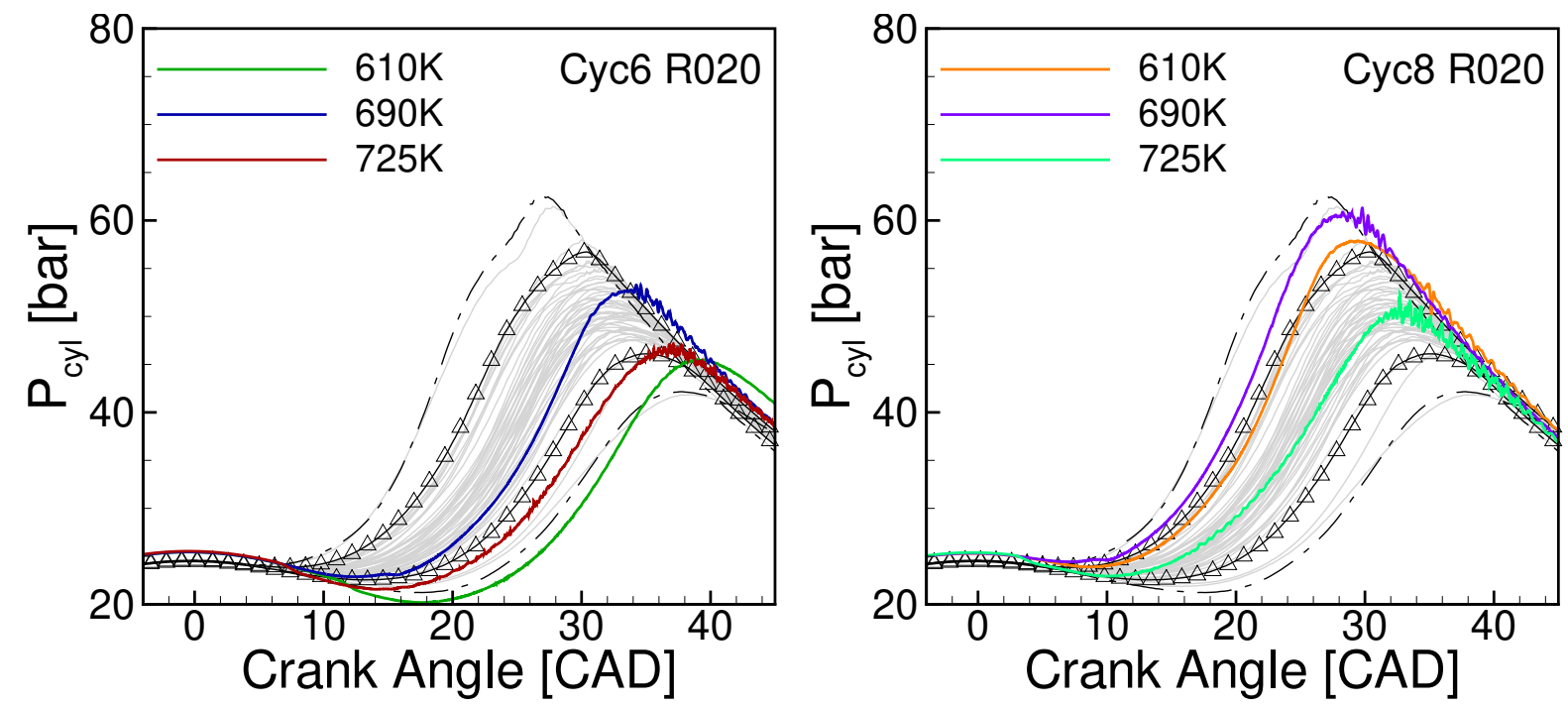

Figure 30: Effects of the exhaust valve temperature on the knock (thin gray lines: 50 cycles cylinder pressure randomly selected from 5000 cycles in the ORNL experiment, black dashed-dotted lines: fastest and slowest cycles in the experiment, black lines with triangles: $5 \%$ and $95 \%$ bounds from 5000 cycles in the experiment, thick colored lines: probed pressure from selected LES cycles).

conditions are summarized in Table ??. More details of the models and the simulation parameters are described below.

\section{Computational Models and Simulation Details}

The FPF method and the laminar-to-turbulent flame transition model described in Sec. 2 [32] are used to described spark-ignited premixed flame propagation. The subfilter flame speed is estimated using the LES version of Peter's model. The model constants used in the sub-filter flame speed model are $b_{1}=9.5$ and $b_{3}=1.8$. The model constants in the laminar-to-turbulent flame transition model are $\eta_{0}=1.5 \mathrm{~mm}$ and $k=10$. As shown below, it turns out that the current model constants overpredict the burning rates in this EGR case.

The spatially-integrated total-enthalpy-based CMC model is used to describe end-gas ignition. The ignition simulation in CMC starts at about -100 CAD relative to combustion TDC. The reduced chemical mechanism for PRF [10,11], which consists of 121 species and 538 reactions, is used. After the validation for the stoichiometric case, we have made several minor modifications to the UDF code for the knock model. Most importantly, the pseudo-enthlapy, which removes the effects that pressure oscillations due to ignition have on the enthalpy fluctuations using the low Mach number approximation, is used as a conditioning variable throughout. For the case described above under the stoichiometric operation with no EGR, at the start of the CMC ignition, enthalpy is used as a conditioning variable until heat release becomes significant. We noticed that the mixture composition stratification due to direct injection makes it difficult to capture temperature fluctuations in the present CMC where the mixture composition is assumed to be homogeneous, when the enthalpy is used as a conditioning variable. The method used for the stoichiometric case is found to work only for very weak composition stratification. Also, at the start of solving the CMC equations, the filtered species mass fractions solved for in CONVERGE are averaged to provide the initial condition for CMC. This allows for considering the residual gas in CMC. The 
mixtures with the equivalence ratio $\phi$ being close to 1 , e.g., $0.9<\phi<1.1$, are considered and the very rich and lean mixtures are excluded when averaging. The overall solution procedure follows one described in Sec. 2.

The spray and other models used are unchanged from those used for the stoichiometric case. The boundary conditions for the wall temperature are also unchanged, and summarized in Table 8. The composition of the exhaust gas is obtained by assuming the complete combustion of the stoichiometric mixture. For the present case, R023, the EGR mass percentage is $15 \%$. The species mass fractions at the intake are calculated accordingly. The computational mesh and its setting for AMR are also unchanged from the stoichiometric case.

\section{$\underline{\text { Results }}$}

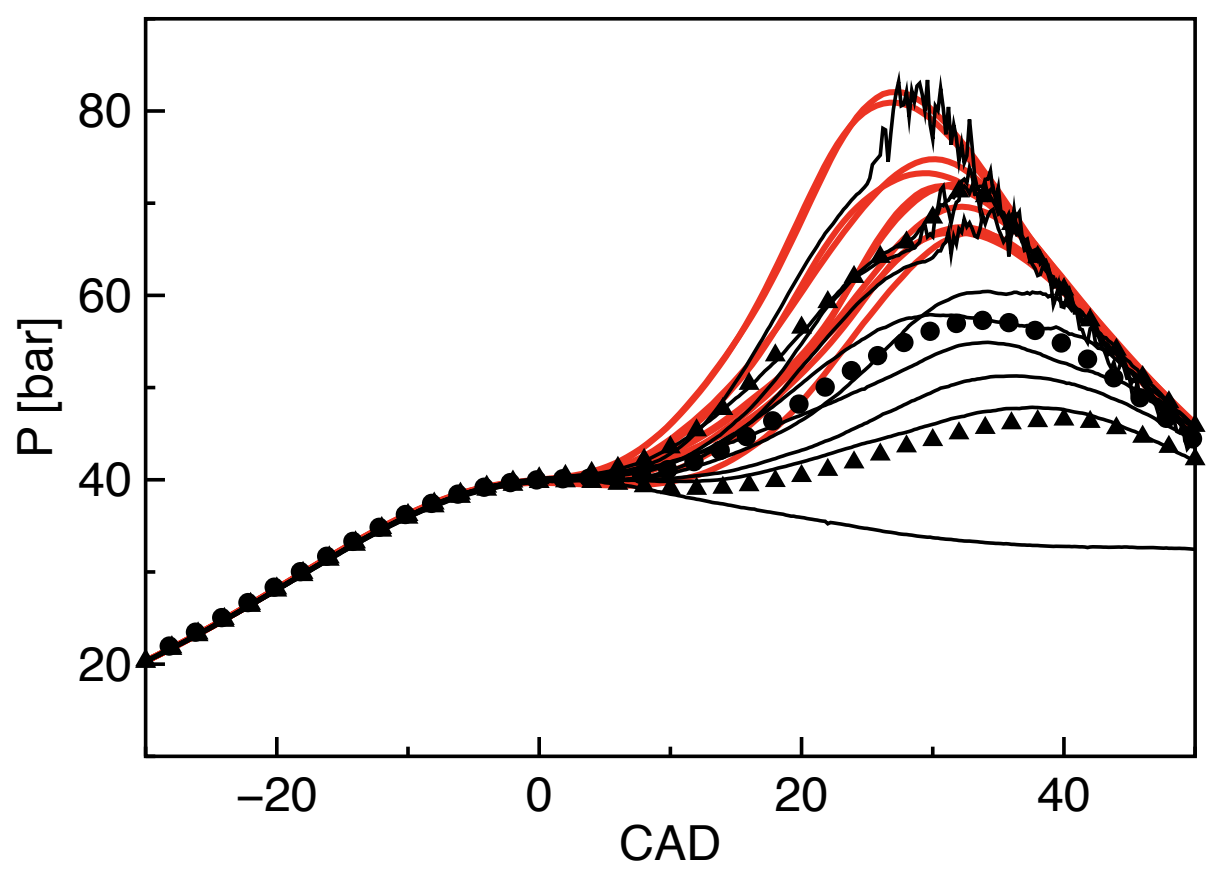

Figure 31: In-cylinder pressure evolution in multi-cycle LES with total-enthalpy-based CMC for a case with EGR (red solid lines: LES, black lines: selected cycles from the experiment including cycles with the maximum and the minimum peak pressure, triangles: 5\% and $95 \%$ bounds from 5000 cycles in the experiment, circles: measured mean pressure).

Figure 31 shows the evolution of in-cylinder pressure. In the experiment, knock is observed for cycles where the peak pressures are high, while it does not happen for slowly burning cycles. For LES, the predicted pressure for 10 cycles is generally higher than the measured one, covering the thermodynamic trajectories that knocking cycles in the experiment pass. However, the present model does not predict knock in this case with EGR. There may be several possibilities that cause the failure of the model in predicting knock, e.g., uncertainties in the wall boundary conditions and associated heat transfer, the reduced chemical mechanism, and the modeling assumptions used in CMC. It is, however, unclear at this point which factor is the most dominant. 


\section{Products}

From the research activities supported by the project, the following papers are published or submitted for publication:

A. Journal Papers:

W. Wang and S. H. Kim. 2019 "Sensible-enthalpy-based conditional moment closure for homogeneous charge compression ignition with temperature inhomogeneity," Flow, Turbulence and Combustion 102, 775-794.

Y. Su and S. H. Kim. 2018 "A consistent, conservative high-order finite difference scheme for low Mach number variable-density flows," Journal of Computational Physics 372, 202219.

Y. Su, D. Splitter and S. H. Kim "Laminar-to-turbulent flame transition and cycle-to-cycle variations in large eddy simulation of spark-ignition engines," submitted.

S. H. Kim and Y. Su "Front propagation formulation for large eddy simulation of turbulent premixed flames," submitted.

B. Conference Papers:

Y. Su, D. Splitter and S. H. Kim, "Predicting cycle-to-cycle variations in a spark-ignition engine using multi-cycle large eddy simulation,” The 11th U. S. National Combustion Meeting Paper, Pasadena, CA, USA, 2019.

W. Wang and S. H. Kim, "Assessment of enthalpy-based conditional moment closure models in predicting ignition of lean and stoichiometric PRF-air Mixtures with temperature inhomogeneity," The 11th U. S. National Combustion Meeting Paper, Pasadena, CA, USA, 2019.

\section{References}

[1] A. Y. Klimenko and R. W. Bilger. Conditional moment closure for turbulent combustion. Prog. Energy Combust. Sci., 25:595-687, 1999.

[2] Wei Wang and Seung Hyun Kim. Sensible-enthalpy-based conditional moment closure model for homogeneous charge compression ignition with temperature inhomogeneity. Flow, Turbulence and Combustion, pages 1-20, 2018.

[3] S. H. Kim. A front propagation formulation for under-resolved reaction fronts. J. Comput. Phys., 285:193-207, 2015.

[4] S. H. Kim and H. Pitsch. Conditional filtering method for large eddy simulation of turbulent nonpremixed combustion. Phys. Fluids, 17:105103, 2005.

[5] S. H. Kim and H. Pitsch. Mixing characteristics and structure of a turbulent jet diffusion flame stabilzed on a bluff-body. Phys. Fluids, 18:075103, 2006.

[6] P. N. Brown, G. D. Byrne, and A. C. Hindmarsh. VODE: a variable-coefficient ODE solver. SIAM J. Sci. Stat. Comput., 10:1038-1051, 1989. 
[7] David L Ropp and John N Shadid. Stability of operator splitting methods for systems with indefinite operators: reaction-diffusion systems. Journal of Computational Physics, 203(2):449-466, 2005.

[8] S. B. Pope. CEQ: A Fortran library to compute equilibrium compositions using gibbs function continuation, 2003.

[9] Thierry Passot and Annick Pouquet. Numerical simulation of compressible homogeneous flows in the turbulent regime. Journal of Fluid Mechanics, 181:441-466, 1987.

[10] Pinaki Pal, Yunchao Wu, Tianfeng Lu, Sibendu Som, Yee Chee See, and Alexandra Le Moine. Multi-dimensional CFD simulations of knocking combustion in a CFR engine. In ASME 2017 Internal Combustion Engine Division Fall Technical Conference, pages V002T06A017-V002T06A017. American Society of Mechanical Engineers, 2017.

[11] Yunchao Wu, Pinaki Pal, Sibendu Som, and Tianfeng Lu. A skeletal chemical kinetic mechanism for gasoline and gasoline/ethanol blend surrogates for engine CFD applications. In International Conference on Chemical Kinetics, Chicago, IL, May, pages 21-25, 2017.

[12] Philipp Schiffmann, David L Reuss, and Volker Sick. Empirical investigation of spark-ignited flame-initiation cycle-to-cycle variability in a homogeneous charge reciprocating engine. Int. J. Engine Res., 19(5):491-508, 2018.

[13] Wei Zeng, Seunghwan Keum, Tang-Wei Kuo, and Volker Sick. Role of large scale flow features on cycle-to-cycle variations of spark-ignited flame-initiation and its transition to turbulent combustion. Proc. Combust. Inst., 2018.

[14] Stéphane Richard, Olivier Colin, Olivier Vermorel, Adlène Benkenida, Christian Angelberger, and Denis Veynante. Towards large eddy simulation of combustion in spark ignition engines. Proc. Combust. Inst., 31(2):3059-3066, 2007.

[15] Norbert Peters. Turbulent combustion. Cambridge university press, 2000.

[16] P. Boudier, S. Henriot, T. Poinsot, and T. Baritaud. A model for turbulent flame ignition and propagation in spark ignition engines. Symposium (International) on Combustion, 24(1):503 - 510, 1992. Twenty-Fourth Symposium on Combustion.

[17] O. Desjardins, G. Blanquart, G. Balarac, and H. Pitsch. High order conservative finite difference scheme for variable density low mach number turbulent flows. J. Comput. Phys., 227:7125 - 7159, 2008.

[18] R. J. Kee, F. M. Rumpley, and J. A. Miller. Chemkin-II: A Fortran chemical kinetics package for the analysis of gas phase chemical kinetics. Technical Report SAND89-9009B, Sandia National Laboratories, 1996.

[19] C. K. Westbrook. Chemical kinetics of hydrocarbon ignition in practical combustion systems. Proc. Combust. Inst., 28:1563-1577, 2000. 
[20] E. Pomraning. Development of large eddy simulation turbulence models. PhD thesis, University of Wisconsin-Madison, 2000.

[21] Rolf D Reitz and R Diwakar. Structure of high-pressure fuel sprays. SAE Trans., pages 492-509, 1987.

[22] Daniel D Joseph, J Belanger, and GS Beavers. Breakup of a liquid drop suddenly exposed to a high-speed airstream. Int J. Multiphas. Flow, 25(6-7):1263-1303, 1999.

[23] Alex B Liu, Daniel Mather, and Rolf D Reitz. Modeling the effects of drop drag and breakup on fuel sprays. SAE Trans., pages 83-95, 1993.

[24] Anthony A Amsden, Peter J O'Rourke, and T Daniel Butler. KIVA-2: A computer program for chemically reactive flows with sprays. Technical Report LA-11560-MS, Los Alamos National Laboratory, 1989.

[25] John L Lumley. Engines: an introduction. Cambridge University Press, 1999.

[26] Le Zhao, Ahmed Abdul Moiz, Sibendu Som, Navin Fogla, Michael Bybee, Syed Wahiduzzaman, Mohsen Mirzaeian, Federico Millo, and Janardhan Kodavasal. Examining the role of flame topologies and in-cylinder flow fields on cyclic variability in spark-ignited engines using large-eddy simulation. Int. J. Engine Res., page 1468087417732447, 2017.

[27] Olivier Vermorel, Stéphane Richard, Olivier Colin, Christian Angelberger, Adlène Benkenida, and Denis Veynante. Towards the understanding of cyclic variability in a spark ignited engine using multi-cycle les. Combust. Flame, 156(8):1525-1541, 2009.

[28] Karine Truffin, Christian Angelberger, Stéphane Richard, and Cécile Pera. Using large-eddy simulation and multivariate analysis to understand the sources of combustion cyclic variability in a spark-ignition engine. Combust. Flame, 162(12):4371-4390, 2015.

[29] Victor Granet, Olivier Vermorel, Corine Lacour, Benoît Enaux, Vincent Dugué, and Thierry Poinsot. Large-eddy simulation and experimental study of cycle-to-cycle variations of stable and unstable operating points in a spark ignition engine. Combust. Flame, 159(4):1562-1575, 2012.

[30] Ömer L. Gülder. Correlations of laminar combustion data for alternative S.I. engine fuels. In West Coast International Meeting and Exposition. SAE International, aug 1984.

[31] C Jiménez, F Ducros, Benedicte Cuenot, and Benoit Bédat. Subgrid scale variance and dissipation of a scalar field in large eddy simulations. Physics of Fluids, 13(6):1748-1754, 2001.

[32] Y. Su, D. Splitter, and S. H. Kim. Laminar-to-turbulent flame transition and cycle-to-cycle variations in large eddy simulation of spark-ignition engines. Int. J. Engine Research, page submitted, 2020.

[33] Rolf D Reitz. Modeling atomization processes in high-pressure vaporizing sprays. Atom. Spray Technol., 3(4):309-337, 1987. 
[34] Peter J O'Rourke and AA Amsden. A spray/wall interaction submodel for the KIVA-3 wall film model. SAE Trans., pages 281-298, 2000.

[35] Antony Misdariis, Olivier Vermorel, and Thierry Poinsot. Les of knocking in engines using dual heat transfer and two-step reduced schemes. Combustion and Flame, 162(11):43044312, 2015.

[36] A. Robert, S. Richard, O. Colin, L. Martinez, and L. De Francqueville. LES prediction and analysis of knocking combustion in a spark ignition engine. Proc. Combust. Inst., 35:29412948, 2015. 